\title{
A STUDY OF CHROMIUM CARBIDE PRECIPITATION AT INTERPHASE BOUNDARIES IN STAINLESS STEEL WELDS
}

\author{
Christopher Fowler Willis
}

\begin{abstract}
Materials and Chemical Sciences Division
National Center for Electron Microscopy, Lawrence Berkeley Laboratory University of California, Berkeley, CA. 94720
\end{abstract}

This work was supported by the Office of Defense Waste and Transportation Management and the Director, Office of Energy Research, Office of Basic Energy Sciences, Material Sciences Division of the U.S. Department of Energy under Contract No. DE-AC0376 SF00098. 


\section{Table of Contents}

Acknowledgements

List of Tables

List of Figures

v

1. Background

1.1. Introduction

1.2. 308 Stainless Steel Weld Microstructures

1.3. Sensitization of Duplex Steel

1.3.1. Qualitative Behavior

1.3.2. Mechanism of Carbide Precipitation

1.3.3. Dependence of Resistance to Sensitization upon $N_{L}{ }^{\alpha-\gamma}$

1.3.4. Sensitization of 308 Stainless Steel Welds

1.4. Chromium Carbide Nucleation and Growth in Stainless Steels

1.4.1. Precipitation in Austenitic Stainless Steel

1.4.2. Precipitation in Duplex Stainless Steel

2. Composition Profiles

3. Goals of Research

4. Experimental Procedures

4.1. Welding

4.2. Heat Treatment

4.3. Specimen Preparation

4.4. Microscopy

5. Results

5.1. Carbide Precipitation in Skeletal Ferrite 26

5.2. Meastirament of Chromium Depletion Profiles 27

5.3. Effect of Phase Boundary Misorientation 29 
5.4. Carbide Nucleation Site in Lathy Ferrite

5.5. Phase Boundary Migration in Lathy Ferrite

6. Discussion

6.1. Carbide precipitation in Skeletal Ferrite

6.2. Measurement of Chromium Depletion Profiles

6.3. Effect of Phase Boundary Misorientation

6.4. Carbide Nucleation Site in Lathy Ferrite

6.5. Effect of Phase Boundary Migration in Lathy Ferrite

7. Conclusions

References
29

30

48

49

51

52

53

57

58 


\section{Acknowledgements}

1 would like to acknowledge the technical contributions of several people to this project.

First of all, Dr. Roger Graham of the Center for Solid State Science at Arizona State University, who operated the high resolution AEM and gave invaluable advice on obtaining the composition profiles. Also, from the Center for Solid State Science, Dr. J.K. Weiss, who wrote the data processing software.

Chuck Echer of the National Center for Electron Microscopy operated the 200CX AEM and provided many helpful observations.

Jin Chen of the Department of Materials Science offered his time and experience during the welding operating.

Dr. Erik Kvam operated the $200 \mathrm{CX}$ high resolution microscope and shared his thoughts on many different aspects of this work, as did Dr. V. Radmilovic. Dr. Radmilovic also gave many helpful hints on specimen preparation.

I would also like to thank the Gronsky research group and the Devine research group for their support and encouragement throughout this project, and $K_{6}$ vin Mon for many enlightening conversations.

This work was performed under the auspices of the U.S. Department of Energy, and supported by the Lavrence Livermore National Laboratory under grant No. W-7405-Eng-48 and by the Director, Office of Basic Energy Sciences, Division of Materials Science of the U.S. Department of Energy under contract No. DE-AC03-76SF 00098, whose support is gratefully acknowledged.

I would also like to acknowledge the interest and encouragement of Dr. Virginia Oversby of the Lawrence Livermore National Laboratory.

Finally, a special thanks to Professor Finnie for reviewing this paper, and to Professors Gronsky and Devine for generously giving their time, expertise, and encouragement throughout the duration of this work. 


\section{List of Tables}

Table

Page

IV - 1 Compositions of Welds $\quad 23$

IV-2 Welding Parameters $\quad 24$

V-1 Measured Chromium Depletion Widths : 32

VI.1 Measured and Calculated Values of $W_{\alpha} \quad 55$ 


\section{List of Figures}

Figure

Page

$1-1 \quad \mathrm{Fe}=70 \mathrm{wt} \%$ section of Fe-Cr-Ni Phase Diagram (from [13]) 11

1-2 Optical Micrograph of a) Skeletal ferrite microstructure b) Lathy ferrite microstructure 12

1. 3 TTS diagram for a) Austenitic stainless steel b) Duplex stainless steel 13

1.4 Schematic of chromium composition profile of sensitized phase boundaries 14

1 - 5 Experimental TTS diagrams of a) skeletal ferrite microstructure and b) lathy ferrite microstructure (from [15,26]) 15

2-1 Schematic showing alectron beam spreading in thin foil 19

4-1 Schematic illustrating geometry of welding and sectioning 25

$5-1$ a) Bright field micrograph of skeletal ferrite aged for 24 hours at $550^{\circ} \mathrm{C}$

b) Bright field micrograph of skeletal ferrite aged for 24 hours at $550^{\circ} \mathrm{C}$ with selected area diffraction pattern from austenite

5 - 2 Bright Field Micrograph of skeletal ferrite aged 24 hours at $550^{\circ} \mathrm{C}$ showing faceted carbides (arrowed) $\quad 34$

5-3 Low energy boundaries (arrowed) in a nominally skeletal microstructure $\quad 35$

5 - 4 High Resolution micrograph of a carbide showing growth ledges (arrows) 36

5 - 5 Centered Dark Field Micrograph $(g=111 \gamma)$ showing carbides embedded in austenite following $\alpha \rightarrow \gamma$ transformation at boundary. (sample aged 24 hours at $550^{\circ} \mathrm{C}$ )

5- 6 Chromium composition profile \#1 and corresponding bright field micrograph of area from sample aged for 1.33 hours at $550^{\circ} \mathrm{C}$

5-7 Chromium composition profile \#2 and corresponding bright field micrograph of area from sample aged for 7.75 hours at $550^{\circ} \mathrm{C}$

5 - 8 Chromium composition profile \#3 and corresponding bright field micrograph of area from sample aged for 16.00 hours at $550^{\circ} \mathrm{C}$

5 - 9 Bright field micrograph from weld 2 showing austenite growth twins, and schematics illustrating orientation relationships of twins $\left(\gamma_{a}\right.$ and $\left.\gamma_{b}\right)$ with ferrite

5-10 Bright field micrograph showing precipitation variation between $\gamma_{\mathrm{a}} / \alpha$ and $\gamma_{\mathrm{b}} / \alpha$ boundaries

5-11 Bright field micrograph showing precipitation variation between $\gamma_{\mathrm{a}} / \alpha$ and $\gamma_{b} / \alpha$ boundaries 
5-12 Bright field micrographs of low energy $\alpha / \gamma$ boundary a) edge-on

b) At large tilt angle, showing high aspect carbide morphology

5 - 13 Bright field micrograph of $\alpha / \gamma$ boundaries in lathy ferrite (weld 3) showing two sets of interface defects

5-14 Bright field micrographs of lathy ferrite (weld 3) aged for 24 hours at $550^{\circ} \mathrm{C}$

5-15 Bright field micrographs of lathy ferrite (weld 3) aged for a) 6 hours and b) 24 hours at $600^{\circ} \mathrm{C}$

6-1 Schematic illustrating growth ledges as possible origin of uneven defect spacing 


\section{Background}

\subsection{Introduction}

Sensitization is a deleterious process which can occur in stainless steels. It is caused by grain boundary or phase boundary precipitation of chromium carbides and the resulting formation of a chromium depleted zone adjacent to these boundaries. The carbides in question actually have the composition. $(\mathrm{Cr}, \mathrm{Fe})_{23} \mathrm{C}_{6}$ (usually written $\mathrm{M}_{23} \mathrm{C}_{6}$ ), and precipitate in the temperature range $450-900^{\circ} \mathrm{C}$. Since a minimum chromium content is required to maintain the passive film necessary for resistance to electrochemical attack, the result of chromium depletion is that the steel becomes "sensitized" to possible intergranular corrosion.

Sensitization often occurs as a result of welding operations. The material close to the fusion line reaches temperatures within the sensitization range. This region is called the heat affected zone (HAZ). Since many welds are multi-pass welds, the actual weld bead of one pass may lie in the heat affected zone of the next pass. The weld bead of the first pass might therefore be sensitized. Furthermore there are applications where welds will be exposed to sensitizing temperatures for very long periods of time, such as welded labels on retrievable nuclear waste containers. For these reasons the sensitization behavior of the actual weld-bead microstructures must be understood.

It has jeen known for many years that duplex stainless steels (steels with both ferrite and austenite phases present at room temperature) have superior resistance to intergranular corrosion [1-3]. A model has been proposed to explain the sensitization behavior of these alloys [4-6]. This work will be concerned with testing the validity of aspects of this model using transmission electron microscopy and further understanding of the sensirization process in duplex stainless steel welds.

\subsection{Stainless Steel Weld Microstructures}

The alloy investigated in this work was 308 stainless steel. In the as-welded condition this is a duplex steel. Two-phase stainless steels possess superior intergranular corrosion resistance, the reasons for which will be discussed in section 1.3. The principal constituents of 308 stainless steel are $\mathrm{Fe}, \mathrm{Cr}$, and $\mathrm{Ni}$. Carbon is also present of course, as well as small amounts of other elements (Mn, Mo, Si, N, P, S). The 308 ASTM definition covers a range of compositions however [7]. Because slight changes in composition can have pronounced effects on the microstructure of 308 welds, - it is useful to describe the alloy by its "chromium to nickel equivalence" (abbr. 
$\{\mathrm{Cr} / \mathrm{Ni}\}$ eq $)$ which is the ratio of the ferrite stabilizing elements to the austenite stabilizing elements in the alloy.

The chromium equivalence, (Cr)eq, is the sum of the weight percents of those elements which are ferritizers (those which stabilize the bcc phase), each weighted by their tendency to promote ferrite in steel. The nickel equivalence is defined in the same way but for austenitizers. The $(\mathrm{Cr} / \mathrm{Ni})$ eq value is obtained by taking the ratio $(\mathrm{Cr})$ eq/(Ni)eq. The various weighting factors are as follows;

$$
\begin{aligned}
& \text { (Cr)eq }=(1 \times[\mathrm{Cr}])+(1.5 \times[\mathrm{Si}])+(1 \times[\mathrm{Mo}]) \\
& \text { (Nii)eq }=(1 \times[\mathrm{Ni}])+(0.5 \times[\mathrm{Mn}])+(30 \times[\mathrm{C}])+(30 \times[\mathrm{N}])
\end{aligned}
$$

To simplify the discussion of 308 stainless steel microstructures it is convenient to refer to the $\mathrm{Fe}-\mathrm{Cr}-\mathrm{Ni}$ ternary phase diagram. It should be understood, however, that the actual positions of the invariant lines may vary from those indicated by this phase diagram since the actual steel contains other constituents which, as mentioned above, affect the relative stabilities of the ferrite and austenite phases.

Figure $1-1$ is a $\mathrm{Fe}=70$ wi\% section of the ternary $\mathrm{Fe}-\mathrm{Cr}-\mathrm{Ni}$ phase diagram [13]. The approximate location of 308 stainless, marked by a dashed line, is misleading. This alloy has a range of compositions as mentioned above. This composition range is roughly from the nickel side of the pseudo-eutectic point (point a in Fig. 1-1) to the point where $\mathrm{Cr} \approx 21 \mathrm{wt} \%, \mathrm{Ni} \approx 9 \mathrm{wt} \%$. The $\delta$ phase in Figure $1-1$ is referred to in this paper as the $\alpha$ phase. Note the curvature of the $\gamma$ solvus and $\alpha$ solvus lines to greater $(\mathrm{Cr} / \mathrm{Ni})$ eqs as the temperature decreases. This indicates that 308 stainless steel of higher ( $\mathrm{Cr} / \mathrm{Ni}$ )eq will undergo an $\alpha \rightarrow \gamma$ transformation at a lower temperature.

Different researchers have studied the microstructures of duplex stainless steel welds [8-15]. At leas! five different microstructures have been distinguished for slightly different composition ranges. This work deals with only two of these microstructures, the skeletal ferrite and lathy ferrite microstructures, which have been studied in detail by Menendez, Chen, and Devine [15]. The origin of these microstructures are as follows;

a) Skeletal ferrite.

308 stainless steel welds with $(\mathrm{Cr} / \mathrm{Ni})$ eq values between 1.43 and 1.68 have a skeletal ferrite microstructure. This microstructure is the result of two phase transtormations which occur as the weld cools from the liquid phase.

The first phase transformation is of course solidification. The weld solidifies as primary ferrite by a cellular mechanism $[12,13,16-18]$. As the cells grow, solute 
partitioning occurs, the ferrite becoming enriched in chromium and the liquid becoming enriched in nickel. When solidification is nearing completion austenite nucleates in the remaining liquid to form intercellular austenite.

As the weld continues to cool a second phase transformation occurs. This is the $\alpha \rightarrow \gamma$ solid state transformation which occurs when the ferrite solvus is crossed, as can be seen by referring to Figure 1-1.

Austenite growth occurs by an equiaxed mechanism [10,12]. In other words the growth rate is roughly the same in all crystallographic directions. There is no tendency for partially coherent, low energy a/y interfaces to be maintained during growth, and such interfaces in the final microstructure are the exception.

For normal cooling rates, the amount of ferrite remaining after room temperature is reached is $\approx 5$ vol\% [12] for these compositions $(1.43-1.68$ $\{\mathrm{Cr} / \mathrm{Ni}\}$ eq). The retained ferrite occupies the cores of the original cells and is therefore termed skeletal ferrite. An example of this morphology is shown in the optical micrograph (Figure 1-2a).

b) Lathy Ferrite.

308 stainless steel welds with $(\mathrm{Cr} / \mathrm{Ni})$ eq values within the range 1.79-2.2 have a lathy morphology.

Again solidification yields a cellular ferrite microstructure. Because of the high chromium content no inter-cellular austenite is formed however [15].

As can be seen by referring to Figure 1-1, the ferrite solvus line is crossed at lower temperatures at these compositions than is the case for welds with skeletal ferrite. As a consequence of the lower temperature regime in which the $\alpha \rightarrow \gamma$ transformation occurs, the nucleation and growth mechanism is a "widmanstatten-like" mechanism [9]. That is, the austenite phase nucleates with an orientation relationship with the ferrite (e.g. a Kurjumov-Sachs relationship [19]) and grows such that the partially coherent, low energy interfaces are maintained. The resulting morphology is a lathy morphology, with the long sides of the laths being partially coherent. It has been shown that the growth of the austenite into the ferrite occurs by a ledge mechanism [20]. An example of this microstructure is shown in the optical micrograph in Figure $1-2 b$.

There has been some debate as to whether the lathy $\alpha \rightarrow \gamma$ phase transformation is a massive (interface-controlled) transformation or a diffusion-controlled transformation [18,21-23]. In a massive transformation there is no long range diffusion, but only short range diffusion across the phase boundary. The growing phase inherits the composition profile of the unstable phase. If the growth of the $\gamma$ into the $\alpha$ 
were a massive transformation, the $\gamma$ would inherit the a composition profile originating from solidification. Instead, measurements of the $\mathrm{Cr}$ and $\mathrm{Ni}$ proflles of as-welded material, indicate segregation of $\mathrm{Cr}$ and $\mathrm{Ni}$ during the solid state transformation [21]. The transformation must be diffusion controlled therefore. This is the case for both skeletal and lathy ferrite microstructures. For this reason the nucleation and growth of the austenite in the lathy ferrite microstructure is said to be "widmanstatten-like" rather than an actual widmanstatten mechanism which would be interface controlled.

Because of the higher $(\mathrm{Cr} / \mathrm{Ni})$ eq, the volume percent of ferrite in the final weld microstructure is $=15 \mathrm{vol} \%$ [12], which is greater than in the skeletal ferrite microstructure. However, the distribution of the ferrite is uneven. There are relatively large areas of untransformed ferrite, as well as very narrow areas of ferrite retairied between the austenite laths which inherit the lathy morphology and give the microstructure its name.

Of course there is no single composition at which the microstructure changes from skeletal to lathy, ratner there is a gradual transition. At $(\mathrm{Cr} / \mathrm{Ni}) \circ \mathrm{Oq}=1.68$ the microstructure is mostly skeletal.. At $(\mathrm{Cr} / \mathrm{Ni})$ eq $=1.79$ the microstructure is mostly lathy. In the composition range between these two values both microstructures are present in varying proportions.

Finally, it should be mentioned that the welding process parameters. such as torch speed and energy input, are found to have a rather small effect on the final microstructure when compared with the effects of composition [15].

\subsection{Sensitization of Duplex Steels}

\subsubsection{Qualitative Behavior}

It has been known fo. 60 yrs that duplex stainless steels have greater resistance to sensitization than single phase austenitic stainless steels [1-3].

A Time-Temperature-Sensitization (TTS) diagram illustrates the kinetics of the sensitization process. Figure 1.3 is a schematic TTS diagram comparing the kinetics of an austenitic and a duplex stainless steel.

As can be seen from the diagram, the behavior of the duplex steel differs from that of the austenitic steel in two respects:

1) A faster sensitization start time $\left(t_{s}\right)$

2) A faster healing time ( $\left.t_{h}\right)$ 
Healing is the replenishment of the chromium depleted region by chromlum diffusing in from the bulk.

At $550^{\circ} \mathrm{C}$ in Figure 1-3, the duplex steel is sensitized after $\approx 2$ hours whereas the the austenitic steel is not sensitized until $\approx 20$ hours. However, the diplex steel is healed after $\approx 200$ hours in this example while the austenite in not healed until thousands of hours.

If is the rapld heaing of duplex stainless steels which makes their resistance to intergranular corrosion superior to that of austenitic stainless steels $[4,5]$.

\subsubsection{Mechanism of Carbide Precipitation}

Devine has developed a model for the mechanism of carbide precipitation which explains the sensitization behavior of duplex stairiless steel (specifically 308 stainless steel) described above [4-6]. This model is as follows;

The rate determining step in the formation of carbides is the diffusion of chromium to available nucleation sites. Since the diffusivity of chromium is higher in the bcc ferrite phase, carbides form more rapidly at ferrite boundaries. These boundaries are almost exclusively $\alpha / \gamma$ boundaries since the ferrite is present as isolated grains. Furthermore the carbon content of ferrite is low, so the reaction at the $\alpha / \gamma$ boundary is mostly between chromium from the ferrite and carbon from the austenite.

Most of the chromium in the carbides is contributed by the ferrite, however a small amount is contributed by the austenife adjacent to the interface. The resulting chromium profiles across the $\alpha / M_{23} C_{6}$ and $\gamma / M_{23} C_{6}$ interfaces are shown in the schematic (Figure 1-4). Wa and $W \gamma$ refer to the widths of the depleted zones on either side of the carbide. These profiles can be approximated by the well known solution of the differential equation describing diffusion in a semi-infinite medium [5,25]:

$$
\frac{x_{o r}-x_{c r}^{\prime}}{x_{c r}^{b}-x_{c r}^{1}}=\operatorname{erf}\left[\frac{w}{2 \sqrt{D t}}\right]
$$

and since

$$
\operatorname{erf}\left[\frac{1}{2}\right]=\frac{1}{2}
$$

Then when

$$
\frac{x_{c r}-x_{c r}^{\prime}}{x_{c r}^{b}-x_{c r}^{\prime}}=\frac{1}{2}
$$




$$
\frac{W}{2 \sqrt{D t}}=\frac{1}{2}
$$

and

$$
W=\sqrt{D t}
$$

The ratio of the depletion widths - W $\alpha / W \gamma$ - will therefore scale with the square root of the ratio of the diffusivities of chromium in these two phases. That is:

$$
\frac{W_{\alpha}}{W_{\gamma}}=\sqrt{\frac{D_{\alpha}}{D_{\gamma}}}
$$

Since the diffusivity of the chromium in the fcc lattice is low, the depletion width in this phase will be narrow. The depleted zone in the austenite is also deeper, since the equilibrium value of chromium at the $\gamma / M_{23} C_{6}$ interface $\left(X_{C r}\right)^{\gamma}$ is lower than at the $\alpha / M_{23} C_{6}$ interface. Because the depletion on the ausitenite side of the interface is natrow, it can be rapidly replenished with chromium from the bulk. The depletion width in the ferrite, $W \alpha$, is wider, but since the diffusivity of chromium is higher in this phase, it too is rapidly replenished. This explains the rapid healing of $\alpha / \gamma$ bouncaries mentioned above.

In order for a boundary to be sensitized, there must be a continuous chromium depleted zone along the boundary [24]. This does not require continuous carbide coverage. Because the phase boundary is a fast diffusion path for chromium atoms, much of the phase boundary adjacent to the carbide is reduced to the carbide interface equilibrium cornposition ( $x_{a}$ ) [24]. To form a continuous chromium depleted zone along the phase boundary, therefore, all that is required is a carbide spacing sufficiently small for the depleted zones from adjacent carbides to overlap.

Finally, a sensitized boundary will not be subject to intergranular corrosion until the chromium depleted zone obtains a minimum critical width. This minimum critical width is that required for species in the aqueous solution to penetrate down the boundary $[6,24]$.

\subsubsection{Dependence of resistance to sensitization upon $N_{L} \alpha-\gamma$}

A quantitative expression has been developed by Devine [5] which indicates the amount and distribution of $\alpha / \gamma$ buundary area necessary for rapid healing and immunity to sensitization.

As mentioned above, chromium depletion along $\alpha / \gamma$ interfaces is rapidly replenished. This is not the case for chromium depletion at $\gamma / \gamma$ boundaries. For rapid 
healing to occur, therefore, the carbon level in the austenite must reach the solubility limit before carbides begin to precipitate at $\gamma / \gamma$ boundaries. This in turn requires:

a) a sufficient amount of $\alpha / \gamma$ boundary area to tie up all of the available carbon at these boundaries.

b) a suitable distribution of $\alpha / \gamma$ boundaries such that all carbon atoms have an available $\alpha / \gamma$ boundary to diffuse to.

The parameter which accounts for both of the above criteria is $N_{L} \alpha-\gamma$ which can be measured metallographically [5]. That is, in order for the material to heal rapidly there must be a critical $N_{L} \alpha-\gamma$ value. The critical value will be a function of the carbon content, since for higher carbon contents more interfacial $\alpha / \gamma$ area is needed to accommodate it as carbide.

If the $N L^{\alpha-\gamma}$ value is high enough, the material will be immune to sensitization. This means that the rarbon in the austenite reaches its solubility limit before the chromium depleted ugion obtains the minimum critical width necessary for intergranular corrosion.

\subsubsection{Sensitization of 308 stainless steel welds}

As discussed in the first section of this work, the microstructure of a 308 stainless steel weld depends on its composition. Since the different 308 microstructures will have different $N_{L} \alpha-\gamma$ values, their sensitization kinetics will also vary $[5,15]$. Menendez, Chen and Devine [15] studied the sensitization of different 308 weld microstructures including the skeletal and lathy microstructures. The mean $N_{L} \alpha-\gamma$ values of these microstructures are $1279 \mathrm{~cm}^{-1}$ for the skeletal ferrite, and $>3000$ $\mathrm{cm}^{-1}$ for the lathy ferrite.

Experimentally determined TTS diagrams for the skeletal and lathy microstructures studied in this work are shown in Figure 1-5. A detailed description of the experimental procedure is given elsewhere $[15,26]$. Basically the samples were heat-treated for the time/ternperatures indicated and then corroded according to ASTM specification A26270 practice $E$ [27].

The very high $N_{L} \alpha-\gamma$ value of the lathy microstructure met the criterion for immunity to sensitization. However from Figure 1.5 it can be seen that the microstructure is sensitized at $550^{\circ} \mathrm{C}$ and $600^{\circ} \mathrm{C}$. However, as discussed above, many of the boundaries in the lathy microstructure are partially coherent, low energy interfaces. Because of their lower energy these boundaries are less favorable for carbide nucleation. An effective $N_{L} \alpha-\gamma$ value was defined as a measure of the amount and distribution of high energy boundaries, which allow sufficient precipitation for a 
continuous chromium depleted zone along the $\alpha \gamma$ interface to be formed [26]. Based on the effective $N_{L} \alpha-\gamma$ parameter, the lathy microstructure no longer met the criterion for immunity to sensitization. The discrepancy in the severity of sensitization between $550^{\circ} \mathrm{C}$ and $600^{\circ} \mathrm{C}$ poses a problem, however, in that the higher temperature is expected to affect the rate of sensitization (as in the skeletal ferrite example) but not the severity.

\subsection{Chromium Carbide Nucleation and Growth in Stainless Steels}

To understand the factors governing sensitization kinetics, it is necessary to understand the factors governing carbide nucleation and growth. These factors may become evident by examining the carbide crystallography and morphology. Most oi the previous investigations of chromium carbide precipitation concern austenitic stainless steels. These results are relevant to the present study because there are similarities in the crystallography of the carbide precipitation in austenitic and duplex stainless steels. The results from austenitic stainless steels will be summarized below as well as results from previous studies of precipitation in duplex steels.

\subsubsection{Precipitation in Austenitic Stainless Steel}

Chromium carbides having a complex cubic lattice with 92 metal atoms and 24 carbon atoms per unit cell, nucleate at grain boundaries within a temperature range of $450-900^{\circ} \mathrm{C}$ [28]. The carbides nucleate with a cube|lcube orientation relatiunship with one of the grains.

That is,

$$
\begin{aligned}
& (100)_{\gamma} \|(100)_{M_{23} C_{6}} \\
& {[001]_{\gamma} \|[001]_{M_{23} C_{6}}}
\end{aligned}
$$

Because most grain boundaries are high angle boundaries, it follows that the carbides have no orientation relationship with the second grain forming the boundary.

Lewis and Hattersley [28], and Beckitt and Clark [29], showed that certain crystallographic planes and directions, within the cubellcube orientation relationship, have better atomic matching between the carbide and the austenite than others. The best atomic matching occurs on \{111\} planes and along [110] directions within these planes. Based on this data, Singhal and Martin [30] proposed that the carbides nucleate as laths with $\{111\}$ habit planes and $\langle 110\rangle$ axes so as to minimize their surface energy. Upon further growth the carbides form a $\{111\}$ facet with the parallel austenite grain while 
growing preferentially into the high angle grain. Adamson and Martin [31] modified this model slightly by proposing that the carbides nucleate as plates with a $\{111\}$ habit plane.

Both Lewis and Hattersley [28], and Singhal and Martin [30] reported that precipitation is often discontinuous. That is, carbide precipitation is followed by migration of the grain boundary in the vicinity of the carbides. The grain in parallel orientation with the carbide always advances into the high angle grain, the driving force being the reduction of total $\gamma / M_{23} \mathrm{C}_{6}$ surface energy.

\subsubsection{Precipitation in Duplex Steels}

There has been less work done on the crystallography and morphology of chromium carbides in duplex stainless steels, than on austenitic stainless steels. Nevertheless it is well documented that the preferred sites for chromium carbides in duplex stainless steels are at the $\alpha / \gamma$ phase boundaries, and that the carbides form a cubel|cube orientation relationship with the austenite [32-36].

Southwick and Honeycombe [33] studied the precipitation of chromium carbides in a lathy ferrite microstructure. They made the following observations;

- At phase boundaries with an orientation relationship (such as the Kurjumov-Sachs orientation relationship [19]) carbides nucleate with a cubellcube relationship with the austenite, and a K.S. relationship with the ferrite.

- Sometimes the carbides form a habit plane with austenite which is a $\{111\}_{\gamma}$ plane, otherwise they do not exhibit any habit plane.

- Carbide precipitation is associated with ledge risers, intrinsic dislocations, and extrinsic dislocations within the phase boundaries.

An analogous study for skeletal ferrite has not been done. Foulds and Moteff [34] report that in the globular ferrite morphology (a microstructure resulting from the annealing and subsequent sherodization of lathy ferrite), carbides precipitate at $\alpha / \gamma$ boundaries and grow into the ferrite by a "blocky growth" mechanism. In other words there is no orientation relationship between the carbide and the ferrite. Images of carbides in skeletal ferrite show a similar blocky morphology $[35,36]$.

in duplex microstructures with high energy phase boundaries such as the skeletal and globular microstructures, carbide precipitation and growth is anaiogous to 
that in austenitic stainless steels in that the carbides nucleate with a cubellcube relationship with the austenite (the parallel grain in austenitic steel) and grow into the ferrite (the high angle grain) with which they have no orientation relationship. 


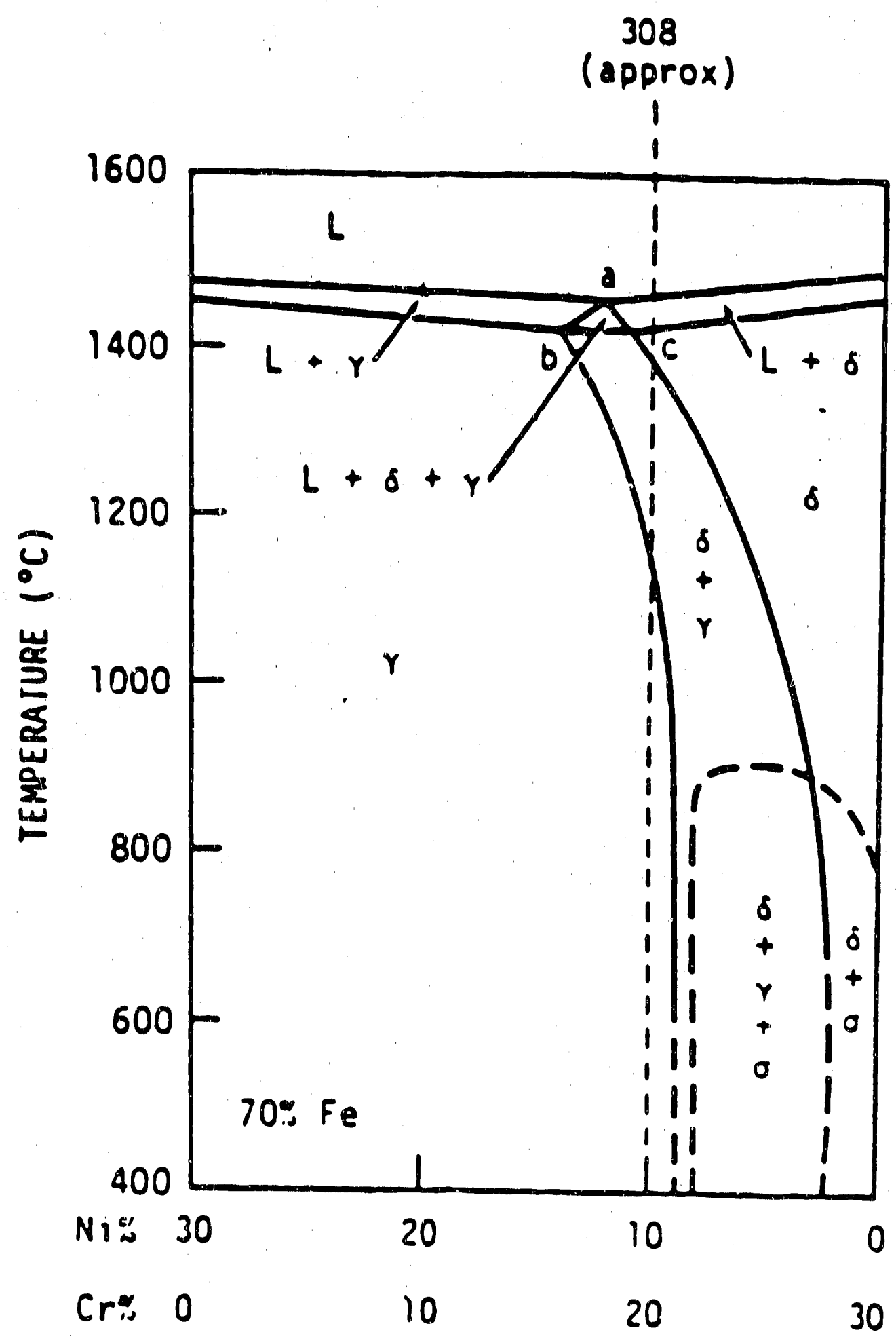

Figure 1.1 

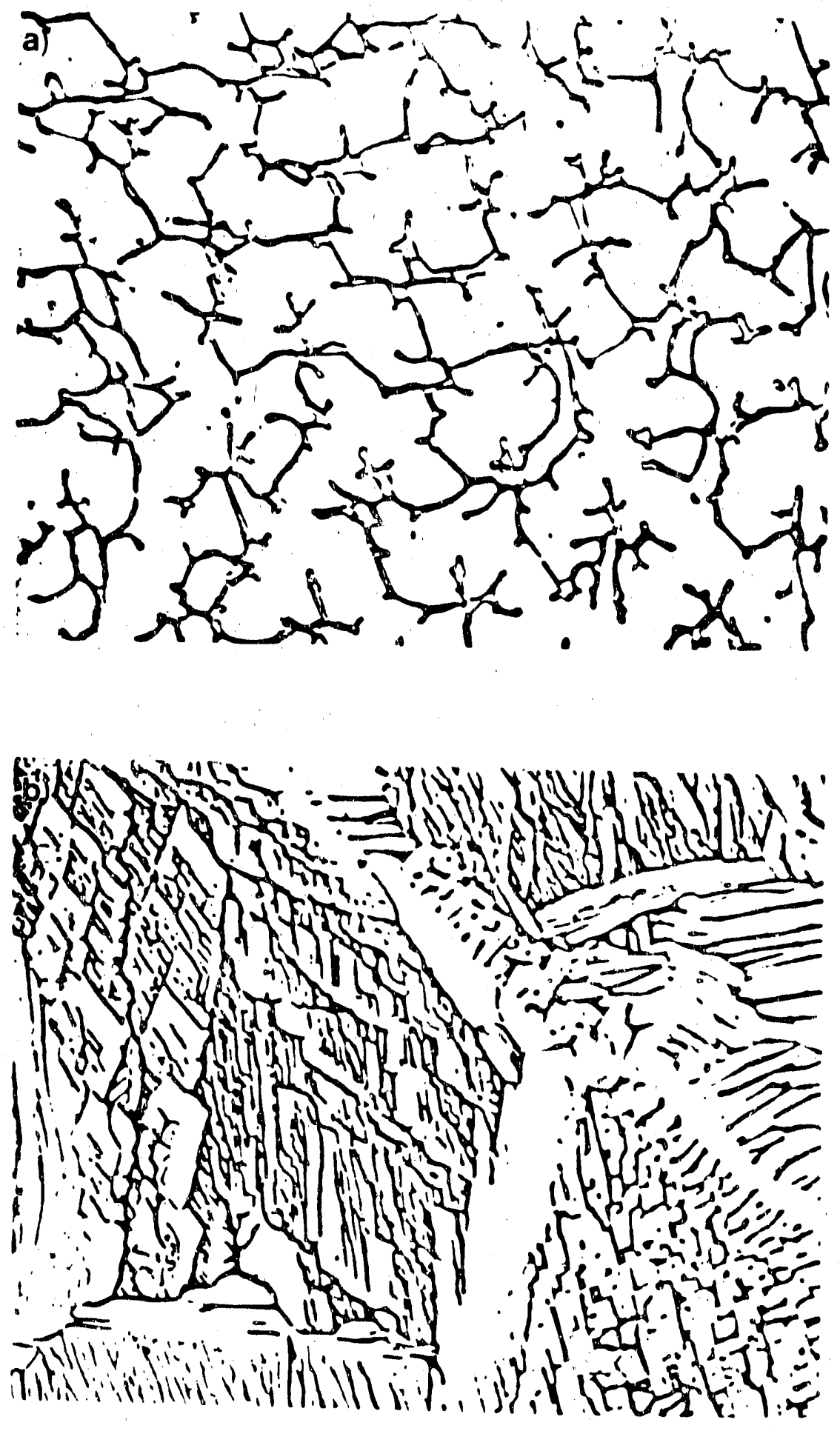

XBB 904-2835 


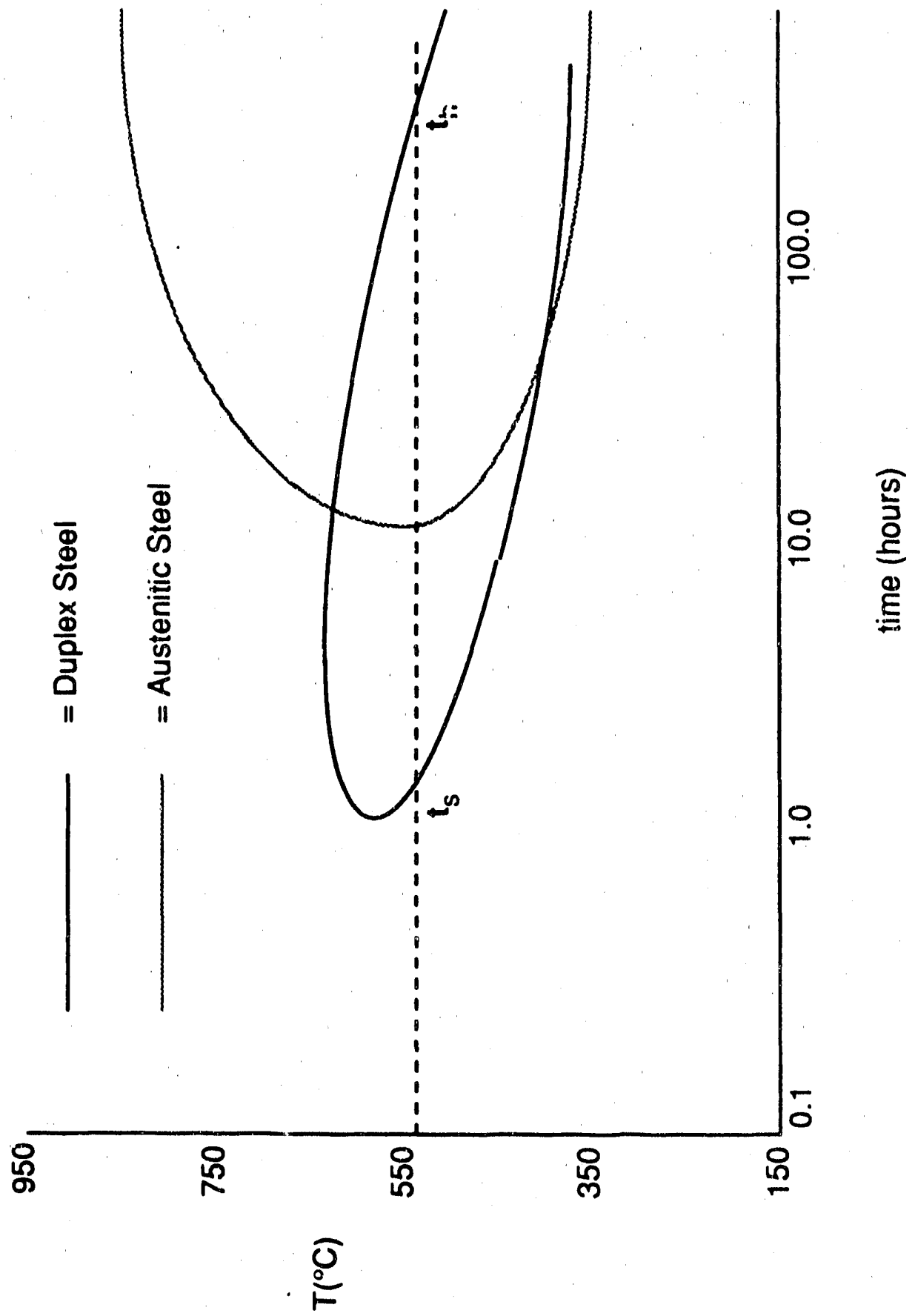

Figure 1-3 


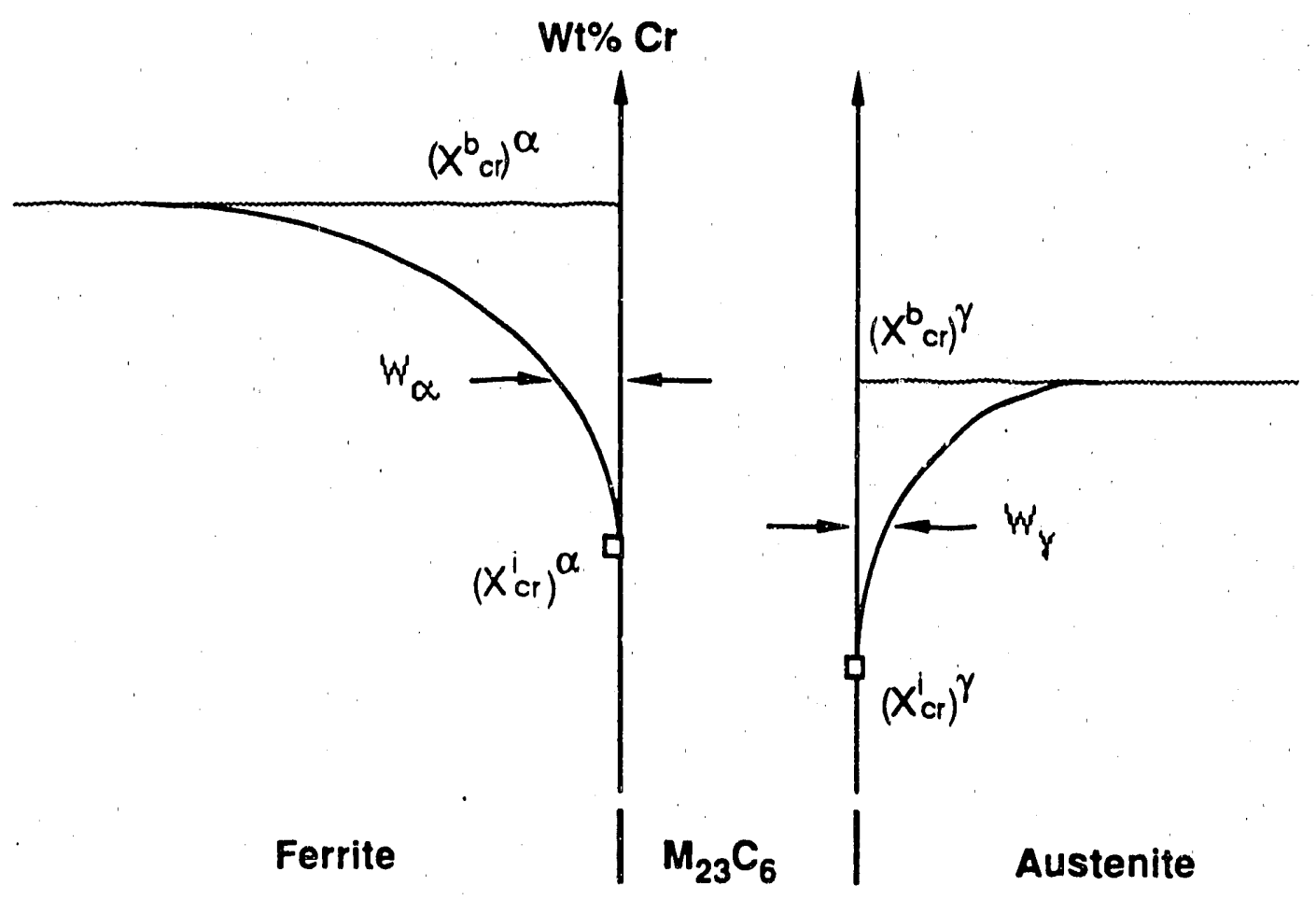

Figure 1-4 
a) Skeletal Ferrite

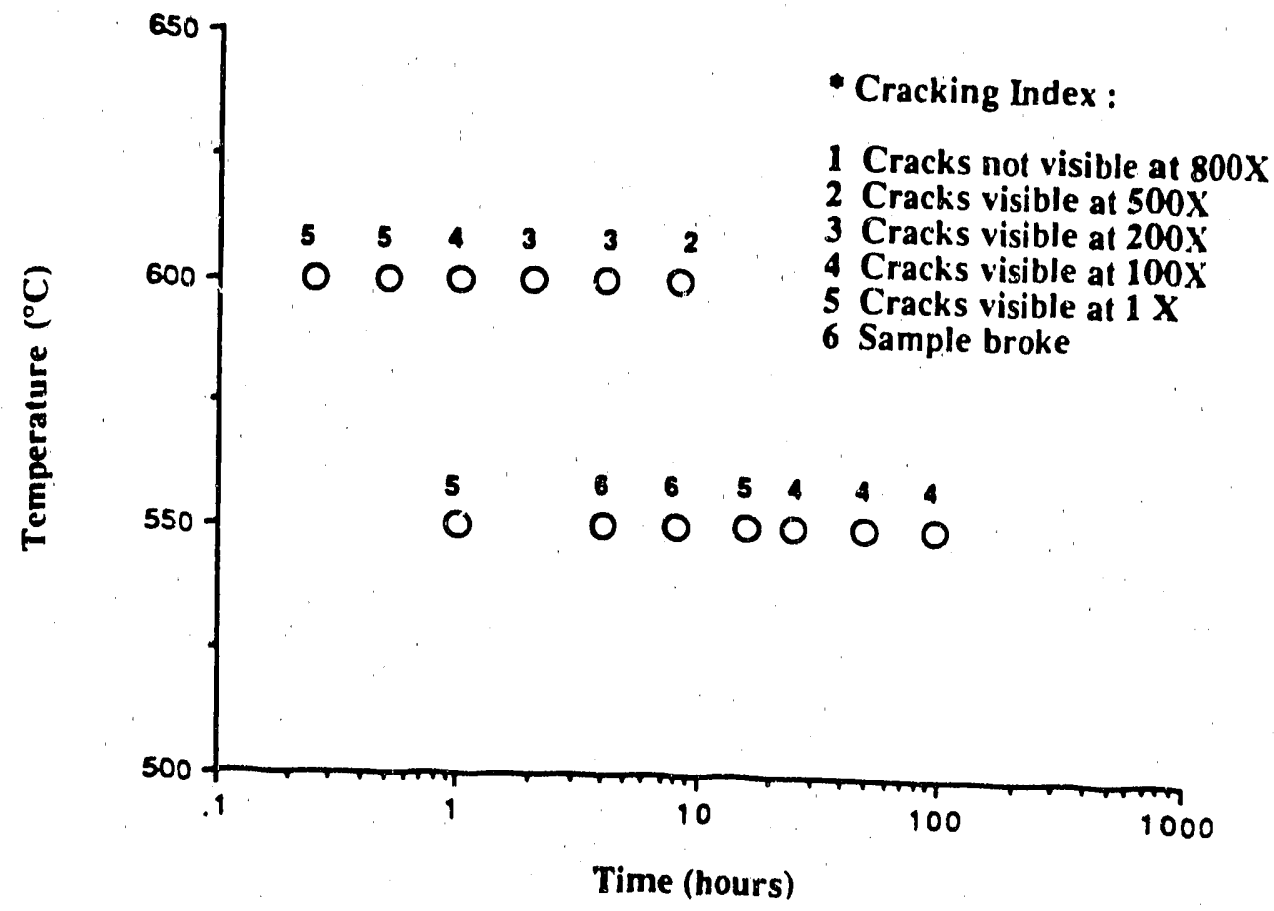

b) Lathy Ferrite

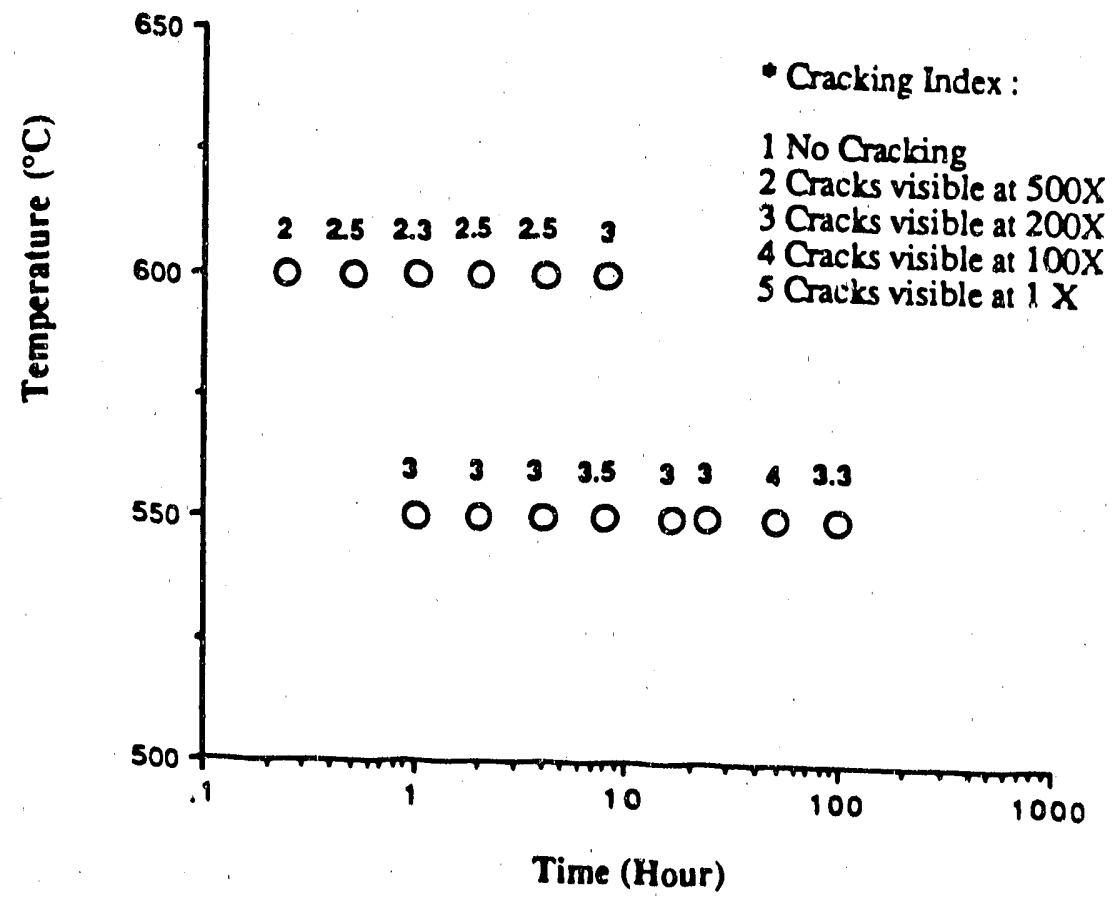

Figure 1-5 


\section{Composition Profiles}

Part of this work involves the measurement of chromium composition profiles across sensitized phase boundaries. This is achieved using a transmission electron microscope equipped with an energy dispersive $x$-ray spectrometer. The limitations of this analytical technique will be discussed briefly here.

The spatial resolution ( $\mathbf{R})$ of this technique is determined by the cioss-sectional area of the excited volume of the specimen. The excited yolume is detarmined by the initial beam diameter (d) and by beam broadening (b) within the sample due to elastic collisions between the beam electrons and the specimen inner shell electrons. This can be written [37]:

$$
R=\sqrt{\left(a^{2}\right)+\left(b^{2}\right)}
$$

Where $\mathbf{R}$ is the effective silameter of the beam at the exit surface of the foil, as shown in Figure 2-1. The beam broadening is described by the following expression [38];

$$
b=\frac{625(Z)\left(p^{1 / 2}\right)\left(t^{3 / 2}\right)}{E_{0}\left(A^{1 / 2}\right)}
$$

where: $\quad Z=$ Alomic number

$\rho=$ Specimen density

$\mathrm{t}=$ Specimen thickness

$E_{0}=$ Accelerating Voltage

$A=$ Atomic weight

From this expression it is clear that $b$ can be decreased by reducing the foil thickness or by increasing the accelerating potential. The rccelerating potential is limited by the microscope used. By using the thinnest areas of the specimen, however, beam broadening can be minimized.

Since the beam diameter also determines the spatial resolution, this parameter must 'e reduced as much as possible. Unfortunately, as the probe is focussed to smaller diameters, the beam brightness (the electron density of the beam) decreases. Since the siatistical error in the number of counts detected is proportional to $1 /\left(n^{1 / 2}\right)$ [39] (where $n$ is the number of counts for a given element), a minimum number of counts must be accumulated at each point for, say, a $3 w t \%$ error. As the probe size is reduced, 
therefore, longer acquisition times must be used for each point in the profile. Since there is always some specimen and beam drift during the acquisition, increasing the acquisition time will effectively reduce the spatial resolution. For this reason, count rate requirements limit the size of the probe which may be used. A microscope equipped with a field emission gun (FEG) can attain higher brightness, allowing probe sizes as low as $10 A$ to be used.

Based on the diffusivity of chromium in the austenite [48], we expect the depletion width in the austenite, $W_{\gamma}$, to be $=115 \mathrm{~A}$ following a 24 hour heat treatment at $550^{\circ} \mathrm{C}$. Very good spatial resolution is needed, therefore, to resolve these profiles. A microscope equipped with a FEG was necessary. The smallest probe which can be used before acquisition times gut prohibitively lung was $20 \AA$. The specimen thickness was $\approx 1000 \mathrm{~A}$ : Based on this information, the beam broadering can be estimated assuming an iron (fcc) specimen.

$$
\text { i.e.: } \quad \begin{aligned}
& Z=26 \\
& \rho=8.5 \mathrm{~g} / \mathrm{cm}^{3} \\
& t=1000 \mathrm{~A} \\
& E_{0}=120 \mathrm{kV} \\
& A=56 \mathrm{~g} \\
& \Rightarrow b=166 \AA \\
& \Rightarrow R=\sqrt{(166)^{2}+(20)^{2}}=167 \AA
\end{aligned}
$$

This calculation yields a value for $b$ which is larger than the expected profile width. Given this result, how accurate will the measured profile width be?

The measured profile is a convolution of the actual profile present in the material, and the shape of the sample volume excited by the beam. Hall et al [38] used computer calculations to perform this convolution and determine the effect of beam spreading on the measured profile. The beam spreading model used was the same as that given above. Qualitatively, the effects of beam spreading are:

1) An increase (decrease) in the minimum (maximum) value measured.

2) A broadening of the measured profile width.

Both of these effects become larger as the ratio of $b$ to the actual profile width increases. The first effect was found to be more severe than the second however. For example their results indicate that for an incident probe diameter of $10 \mathrm{~A}$, and $a$ value of $b \approx 200 \mathrm{~A}$, an 
actual profile $50 \mathrm{~A}$ in width will have a "measured" width of $\approx 60 \mathrm{~A}$. An actual width of $25 \mathrm{~A}$ will have a "measured" width of $40 \mathrm{~A}$. Clearly the error increases as the actual profile width decreases relative to $b$. Nevertheless, even though $b$ is much larger than the actual profile in the second case, the measured profile width is still within $40 \%$ of the actual profile width.

From these calculations of Hall et al [38] it is concluded that the chromium depletion widths measured in this work which are less than $100 \AA$ can not be considered accurate. It is corrent to say, however, that these depletion widths are of the order of magnitude of $10 \AA$. Depletion widths greater than $100 \AA$ are more accurate and, in fact, are most likely "mited by' errors in beam positioning rather than errors due to beam spreading.

The effect of beam spreading does prevent the measurement of chromium profiles across $\gamma / M_{23} C_{6}$ and $\alpha / M_{23} C_{6}$ boundaries. This is due to the high chromium conterll of the carbides which will produce spurious chromium signals at beam locations within the diameter of beam spreading [40]. For this reason profiles were taken from sensitized $\alpha / \gamma$ boundaries adjacent to the carbides. 


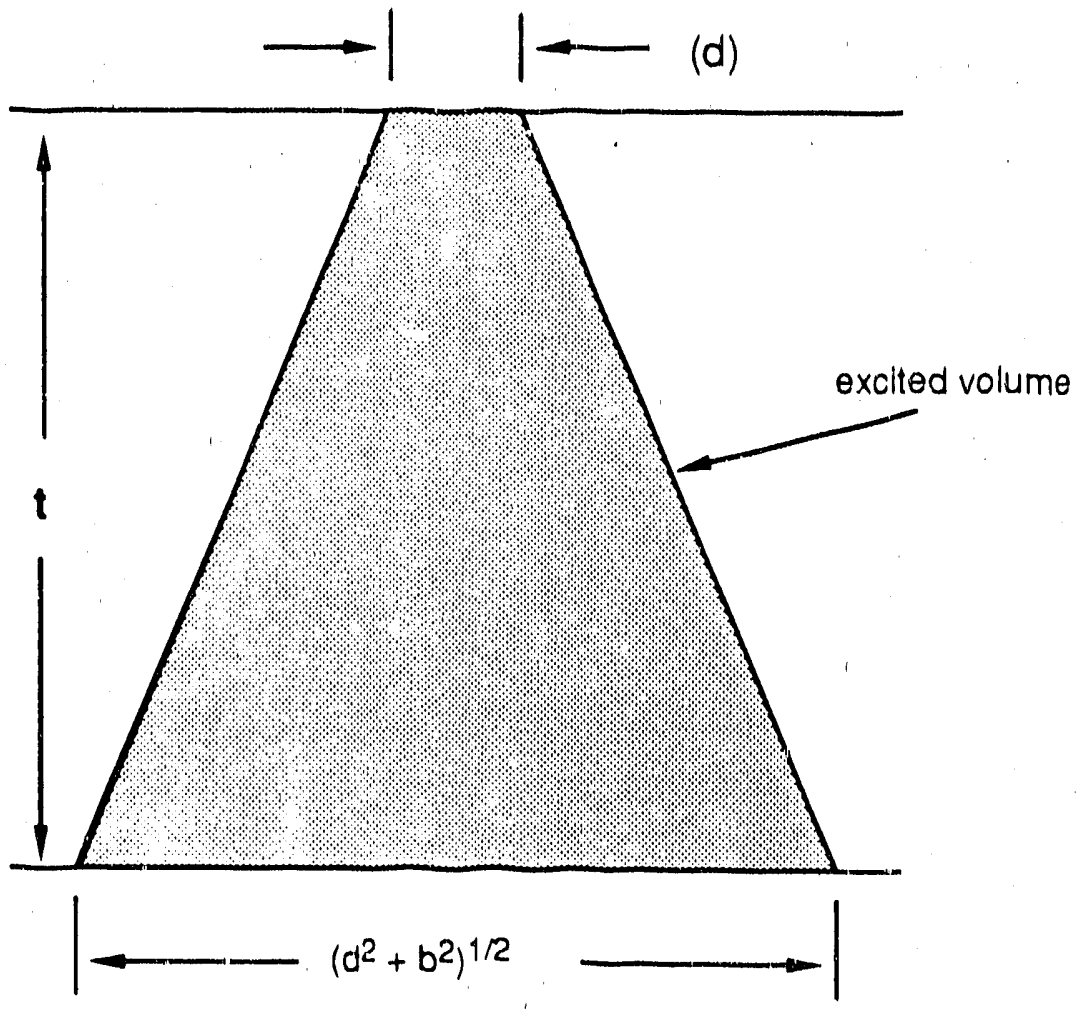

Figure 2-1 


\section{Goals of Research}

This work will address several questions concerning carbide nucleation and growth and the accompanying chromium depletion in 308 stainless steel welds, using electron microscopy techniques. The goals of this research are as follows.

For the skeletal ferrite microstructure:

1) Examine the crystallography and morphology of carbides at $\alpha / \gamma$ interfaces.

2) Determine the relative chromium depletion widths on either side of the $\alpha / \gamma$ interface. The kinetic model predicts relative depletion widths based on the diffusivities of chromium in the two phases, as illustrated in figure 1-4. Because of the experimental difficuliy in measuring chromiurn profiles across the $\gamma / M_{23} C_{6}$ and $\alpha / M_{23} C_{6}$ interfaces, chromium profiles will be obtained instead across a sensitized $\alpha / \gamma$ boundary between two carbides. The measured depletion widths will be compared to those calculated from the diffusivity of chromium.

For the lathy ferrite microstructure:

1) Verify the effect of phase boundary misorientation on the carbide precipitation rate. The lower precipitation rate on many boundaries in this microstructure has been attributed to the low energy of these boundaries. This effect will be studied further.

2) Determine the nucleation sites of the carbides.

Within the partially coherent, low energy phase boundaries in this microstructure there are expected to be preferred nucleation sites on interfacial defects. The nature of these defects will be examined.

3) Explain the sensitization behavior of this microstructure.

An effective $N_{L}^{\alpha-\gamma}$ parameter was defined for this microstructure. This parameter accounts for the sensitization which occurs after aging at $550^{\circ} \mathrm{C}$, however it does not account for the less severe sensitization that occurs after aging at $600^{\circ} \mathrm{C}$. The carbide precipitation will be studied at both of these aging temperatures, with the aim of understanding the sensitization behavior. 


\section{Procedure}

\subsection{Welding}

Three autogenous 308 stainless steel welds having different weiding parameters and compositions were examined in this work.

The welds were made by automaticaily moving a T.I.G. torch across plates, having the dimensions 1 " $\times 4$ " $\times 20$ ", in a direction parallel to the 4 "dimension as illustrated in Figure 4-1. The shielding gas used was Argon.

The nominal composition of each welds is of course the composition of the baseplate upon which the weld is laid down. These compositions are listed in table IV-1. The welding parameters are shown in Table IV-2. The welding parameters are not expected to have a significant influence on the ferrite morphology [15]. The ferrite morphologies of the three welds are those predicted by the $(\mathrm{Cr} / \mathrm{Ni})$ eqs. Weld 1 has a skeletal ferrite morphology, weld 3 has a lathy ferrite morphoiogy, and weld 2 has both skeletal and lathy ferrite areas.

\subsection{Heat Treatment}

Before preparing thin foils for TEM work, the samples were aged at $550^{\circ} \mathrm{C}$ or $600^{\circ} \mathrm{C}$ to obtain sensitized microstructures.

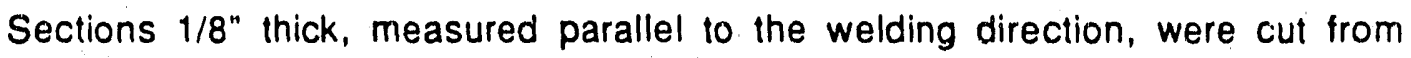
the weld with a diamond saw (shown schematically in Figure 4-1). These were ultrasonically cleaned sequentially in detergent, distilled water, and acetone, and wrapped in Tantalum foil. The sections were then placed Pyrex tubes which were alternately evacuated and back-filled with $\mathrm{N}_{2}$. The tubes were sealed with a residual pressure of $1 / 6 \mathrm{~atm}$. of $\mathrm{N}_{2}$ and placed in a furnace at the appropriate temperalure, for aging times between 1 and 24 hours. After the heat treatments, the tubes were quenched in water.

\subsection{Preparation of TEM Samples}

To obtain TE.M samples, the heat treated sections were first ground down to 3-4 mils with a grinding wheel. Foils thinner than 3 mils produced samples with insufficient structural integrity, while foils thicker than 4 mils yielded samples with insufficient thin area. The foils were next etched to reveal the weld bead. Three $\mathrm{mm}$ disks were punched out of the weld beads and jet polished in 66 vol\% Methanol +33 vol\% Nitric acid solution. The polishing parameters which yielded the best results were a temperature of $-35^{\circ} \mathrm{C}$, and a potential of 12 volts. 
Samples for high resolution work were given an additional ion mill after the jet polish. This was necessary since high resolution worli requires very thin specimen thickness (less than one extinction distance). The ion mill parameters were: time $=2$ hours, angle $=10^{\circ}$, gun voltage $=2 \mathrm{kv}$, gun current $=0.25 \mathrm{~mA} / \mathrm{gun}$. This low gun voltage was chosen to minimize damage to the fcc austenite phase.

\subsection{Microscopy}

Conventional imaging and microdiffraction were performed on a Philips 301 and a Philips 400 microscope.

The analytical microscopy was performed on a Philips 400 ST FEG microscope equipped with a Kevex high-angle EDX delector, in the following manner:

A suitable portion of an $\alpha / y$ phase boundary was chosen which contained two phase boundary $\mathrm{M}_{23} \mathrm{C}_{6}$ carbides separated by a small distance $(\approx 1000 \AA)$. The phase boundary was tilted to a position parallel to the electron beam or "edge-on". A composition profile perpendicular to this boundary, approximately halfway between the two carbides was obtained in the following manner;

The microscope was operated in TEM mode. A 20 A diameter focussed probe was used at each acquisition point. Acquisition times varied from 100 to 300 seconds depending on the chromium count rate. The probe was repositioned approximately every 25 seconds to minimize specimen drift. Compositions were calculated using the thin foil approximation and assuming the signals detected were from the elernents $\mathrm{Fe}, \mathrm{Cr}$, and $\mathrm{Ni}$. The energy resolution of the detector was not high enough to resolve the $\mathrm{Mn}$ peaks, however the amount of $\mathrm{Mn}$ in this alloy is small - $1.5 \mathrm{wt} \%$.

Because of the intense beam some surface ablation occurred under the beam. Comparison of two measurements both made sufficiently far from the boundary to insure that no chromium depletion had occurred, one which sustained damage from ablation and one which showed no damage, indicated that errors caused by ablation are smaller than the errors due to statistical variations in the count rate (represented by vertical error bars on the composition profiles). 


\section{Table IV-1}

Compositions of Welds Studied

(all values in wt\%)

\begin{tabular}{ccccccccc} 
Weld No. & $\mathrm{Cr}$ & $\mathrm{Ni}$ & $\mathrm{Mn}$ & $\mathrm{Si}$ & $\mathrm{C}$ & $(\mathrm{S}+\mathrm{P})$ & $\mathrm{Fe}$ & $(\mathrm{Cr} / \mathrm{Ni}) \mathrm{eq}$ \\
\hline 1 & 20.0 & 12.0 & 1.5 & 0.5 & 0.06 & 0.25 & Bal. & 1.43 \\
2 & 20.66 & 10.8 & 0.99 & 0.5 & 0.17 & 0.16 & Bal. & 1.685 \\
3 & 21.0 & 9.0 & 0.5 & 1.0 & 0.03 & 0.023 & Bal. & 2.22
\end{tabular}


Table IV-2

Welding Parameters Used

$\begin{array}{cccc}\text { Weld No. } & \begin{array}{c}\text { Torch Speed } \\ (\mathrm{in} / \mathrm{min})\end{array} & \begin{array}{c}\text { Heat Input } \\ (\mathrm{KJ} / \mathrm{in})\end{array} & \text { Single/Multi-Pass } \\ 1 & 3 & 69.866 & \text { Single-pass } \\ 2 & 9 & 15.33 & \text { Multi-pass } \\ 3 & 3 & 61.91 & \text { Single-pass }\end{array}$



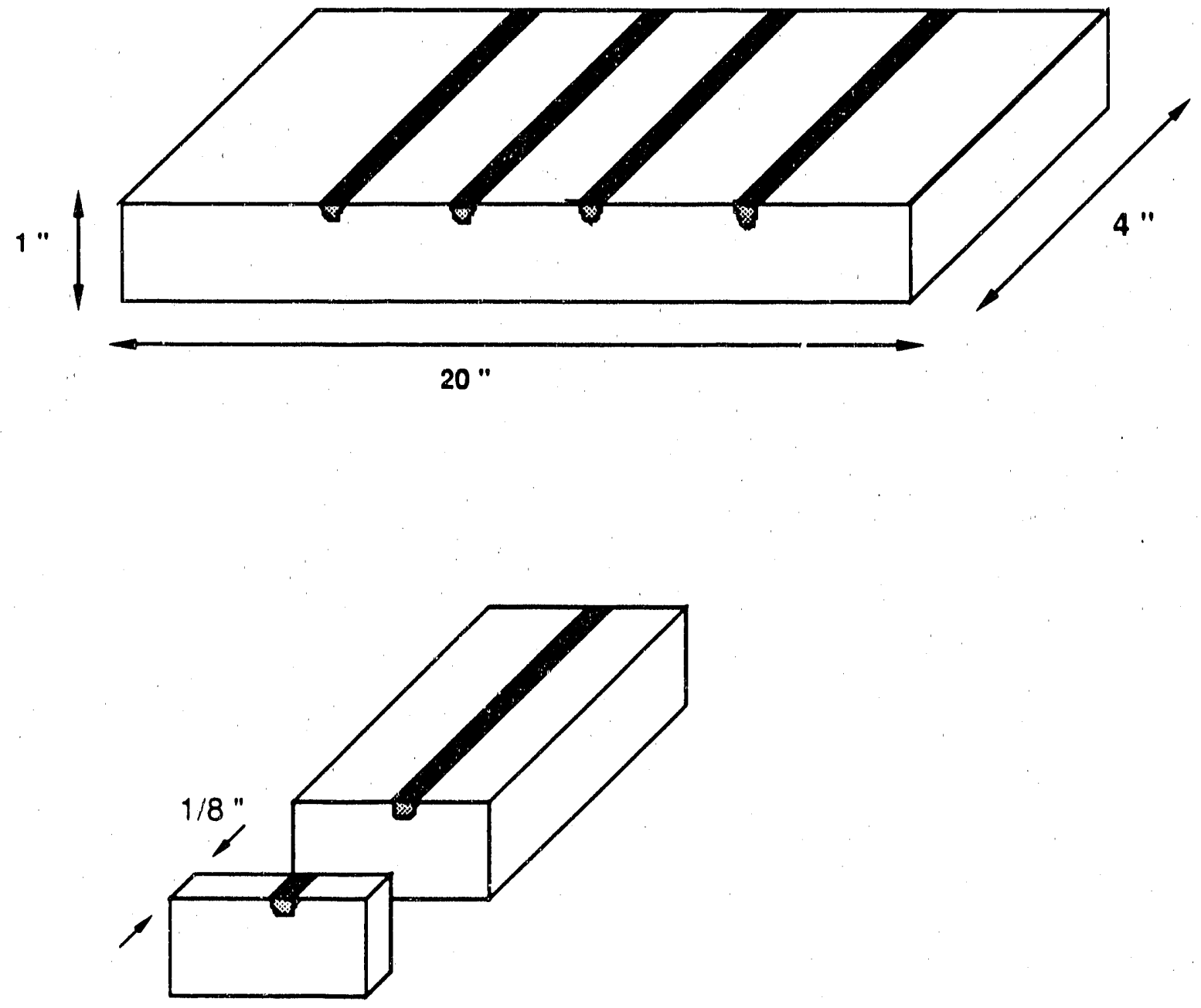

Figure 4-1 


\section{Resulis}

\subsection{Carbide Precipitation in the Skeletal Ferrite Microstructure}

Examples of carbide precipitation at $\alpha / \gamma$ interfaces in the skeletal ferrite microstructure are shown in Figures 5-1a, 5-1b, and 5-2, which are transmission electron micrographs of weld 1 heat-treated for 24 hours at $550^{\circ} \mathrm{C}$. These micrographs are representative of the appearance of a large number of $\alpha / \gamma$ interfaces that were examined. The density of carbides is reasonably constant along the various boundaries although there are some segments of grain boundary that are free of carbide precipitation. Furthermore, the carbides extend into the ferrite phase and are usually faceted. Samples that were heat-treated at $550^{\circ} \mathrm{C}$ for times of $1.33,7.75,16,48$, and 96 hours were also examined by TEM and the results were the same as those reported for samples heat-treated at $550^{\circ} \mathrm{C}$ for 24 hours. That is, most of the $\alpha / \gamma$ interfaces are high energy boundaries (hence lacking a low-index orientation relationship) upon which carbide precipitation occurs fairly uniformly.

Figure 5-1b shows a selected area diffraction pattern corresponding to diffraction from the austenite and interphase carbides of that micrograph. The more intense reflections originate from electron scattering within the austenite while the weaker reflections originate from scattering within the carbides (this was confirmed by placing the objective aperture over a given reflection and observing which phases appeared bright). The orientation relationship, between the austenite and carbides, is a cubel|cube relationship as expected. That is:

$$
\begin{aligned}
& (100)_{\gamma} \|(100) \mathrm{M}_{23} \mathrm{C}_{6} \\
& {[100]_{\gamma} \|[100] \mathrm{M}_{23} \mathrm{C}_{6}}
\end{aligned}
$$

The relative lengths of the lattice parameters can also be determined from the diffraction pattern, i.e. $a_{\gamma}: a_{M_{23}{ }_{2}} C_{6}=3.6 \AA: 10.65 \AA=1: 3$

As mentioned above, diffraction analysis revealed that most of the skeletal ferrite jrains did not possess a low index orientation relationship with the adjacent austenite phase. Exceptions were found however. An example of a ferrite grain with a NishiyamaWasserman orientation relationship $[41,42]$ is shown in Figure 5-3. The $\alpha / \gamma$ boumiries (such as those arrowed in Fig. 5-3) were always planar whenever the two phases formed a low energy interface. Carbide precipitation also occurred on the low 
energy interphase boundaries, albeit with a lower density than along the high energy $\alpha / \gamma$ boundaries.

It was also mentioned above that the interphase carbides grew preferentially into the skeletal ferrite phase and that many of the interphase carbides were observed to be faceted. Trace analysis indicated that most of these facets are $\{111\}$ or $\{100\}$ type facets. Figure $5-2$ is a bright field image showing faceted interphase carbides. The crystallographic planes of those carbide facets which are "edge-on" (parallel to the electron beam) are indexed. Diffraction analysis showed that there were no crystallographic directions in the adjacent skeletal ferrite which were parallel to the facet normals. Thus while a specific orientation relationship existed between the austenite phase and the carbides, none existed between the carbides and the skeletal ferrite phase Nevertheless the carbides growing into the skeletal ferrite phase were highly faceted.

High resolution images were also obtained of faceted carbides. Figure 5-4 shows a high resolution image of a carbide with two $\{100\}$ facets (roughly parallel to the edges of the micrograph). The arrows indicate ledges which are one unit cell in height.

After aging times of 24 hours or more, there was evidence of $\alpha \rightarrow \gamma$ transformation on some boundaries. One such boundary is shown in Figure 5-5. This is a centered dark field micrograph $(g=111 \gamma)$ showing carbides completely embedded in austenite. The even contrast of the austenite phase indicates that the newly formed austenite has grown epitaxially with respect to the original austenite.

\subsection{Measurernent of Chromium Depletion Profiles}

Figures $5-6,5 \cdot 7$, and $5-8$ show chromium profiles obtained from samples of weld 1 that were aged for $1.33,7.75$, and 16 hours at $550^{\circ} \mathrm{C}$. Recall that weld 1 possessed a skeletal ferrite microstructure. The chromium profiles were obtained by the method outlined in section 4.4. The chromium depletion widths on either side of the $\alpha \gamma$ phase boundaries - $W_{\alpha}$ and $W_{\gamma}$ - were examined.

The depletion widths are defined as the point where:

$$
\frac{x_{c r}-x_{c r}}{x_{c r}^{b}-x_{c r}}=\frac{1}{2}
$$

As illustrated in Figure 1-4. 
This admittedly arbitrary definition of $W_{\alpha}$ and $W_{\gamma}$ was selected because it could be measured with relatively little trouble. For example, $X_{c r}$ values measured at locations closer to the interface will have greater errors due to the effect of beam spreading, since the slope of the actual composition profile, described by the diffusion equation given in section 1.3.2., increases as the interface is approached. At points further away from the interface, the composition approaches the bulk composition asymptotically so small uncertainties in composition lead to larger errors in $W$. The definition of $W$ given above was chosen to avoid these errors.

One feature of the chromium profiles which differs from most chromium profiles measured across grain boundaries in single phase austenitic stainless steels, is their relative flatness on the austenite side of the $\alpha / \gamma$ interface. This is most apparent for that profile obtained from the sample heat-treated for 7.75 hours at $550^{\circ} \mathrm{C}$ (profile \#2). This feature does not occur in the portion of the chromium profile obtained in the ferrite phase. The significance of this observation will be described in the discussion.

It is difficult to measure $\left(X_{a}\right)$ because this represents the point at which the error due to beam spreading is most severe [43]. There are two ways in which $\left(X_{c t}\right)$ may be estimated. The composition profile measured perpendicular to the phase boundary can be fit to an error f'nction and the curve extrapolated to $x=0$ (the $\alpha / \gamma$ interface), or a value can be calculated using available thermodynamics data. Because of the small number of data points, the first method is not accurate. In addition, the unexpected flatness of the profile on the austenite side of the interface indicates that the phenomena responsible for the shape of the chromium profile are not fully understood. In the face of this uncertainty the best possible estimate of $\left(X_{\alpha}\right)^{\alpha}$ would be obtained by by the following method. First it is assumed that at the $\alpha / \gamma$ interface the chromium activities in the two phases are equivalent. That is:

$$
\left(a_{c r}\right)^{\alpha}=\left(a_{c r}\right)^{p}
$$

Therefore

$$
\begin{gathered}
\left(x_{c r}^{i}\right)^{\alpha}\left(\gamma_{c r}\right)^{\alpha}=\left(x_{c r}^{i}\right)^{\gamma}\left(\gamma_{c r}\right)^{\gamma} \\
\text { (where } \gamma_{c r}=\text { The chromium activity coefficient) }
\end{gathered}
$$

The relatively flat portion of the chromium profile in the austenite phase is extrapolated to $x=0$ to obtain $\left(x_{\alpha}\right)^{\gamma}$. The activity coefficients are obtained from reference [26]. From these values a good estimate of $\left(X_{c}\right)^{\alpha}$ can be obtained. 
Applying the above method to profile \#3 a value of $\left(X_{\alpha}\right)^{\alpha}=11.2 \mathrm{wt} \%$ was obtained. Given this value, $W_{\alpha}$ can be measured off of the $\mathrm{Cr}$ profiles. The results are summarized in Table V-1.

\subsection{Effect of Phase Boundary Misorientation}

The density of carbides along the $\alpha / \gamma$ boundaries in weld 1 (skeletal ferrite microstructure) was fairly uniform principally because, as described in section 5.1., most of the $\alpha / \gamma$ interphase boundaries were high energy interfaces.

Evidence of variation in the density of interphase carbides with phase boundary misorientation was particularly strong in the case of weld 2, which had a mixed microstructure consisting of skeletal ferrite plus lathy ferrite, and in the case of weld 3 which had a lathy ferrite microstructure.

Figure 5.9 shows a region of weld 2 which has formed growth twins upon solidification. The austenite is designated $\gamma_{a}$ and its twin $\gamma_{b}$. The ferrite, $\alpha$, is also labeled. The orientation relationships between $\gamma_{a}$ and $\alpha$, and between $\gamma_{b}$ and $\alpha$, are indicated by the diffraction patterns in Figure 5-9. It can be seen that the $\gamma_{b}$ and $\alpha$ lattices have two parallel directions within the zone axis. That is:

$$
[\overline{11} \overline{1}]_{\gamma_{b}} \|[0.1 \overline{1}]_{\alpha}
$$

and

$$
[00 \overline{2}]_{\gamma_{b}} \|[\overline{1} 10]_{\alpha}
$$

The $\gamma_{\mathrm{a}}$ and $\alpha$ lattices on the other hand have no parallel directions within this zone axis. It should be mentioned that the orientations shown in Figure 5-9 corresponded to a specimen tilt at which the $\gamma_{a} / \alpha$ and $\gamma_{b} / \alpha$ boundaries were "edge-on". This can be verified by examining Figures 5.10 and 5.11 obtained at the same specimen tilt.

Figures 5.10 and 5.11 show that carbide precipitation occurs on the high energy $\gamma_{a} / \alpha$ boundary, but not on the low energy $\gamma_{b} / \alpha$ boundary.

\subsection{Carbide Nucleation site in Lathy Ferrite}

As discussed in section 1.2, the lathy ferrite microstructure is composed of many low energy $\alpha / \gamma$ interfaces. The carbide density on these boundaries is lower than on the skeletal $\alpha / \gamma$ boundaries presumably due to the existence of fewer nucleation sites. The 
nature of the nucleation sites which do exist on these boundaries was examined in this section.

Figure 5-12a shows a phase boundary, labeled A, which is actually a lows energy

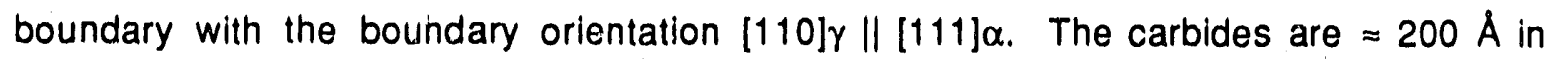
width. When the phase boundary is tilted such that it forms a large angle with the incident electron beam (Figure 5-12b), the distribution of the carbides through the thickness may be observed. Many of the carbides can be seen to run right through the foil. Since the foil is $\approx 1500 \AA$ thick, these carbides have a high-aspect, lenticular morphology. This morphology was prevalent in most of the low energy boundaries observed. For comparison the carbides at the high energy boundary $B$ (in the same micrograph) have an equiaxed morphology. These observations suggest that the carbides on the low energy boundary $A$ are associated with linear interface defects. These defects could be intrinsic dislocations, extrinsic dislocations, or ledge risers. In this example no defects unassociated with carbides were observed.

Figure 5-13 shows two $\alpha / \gamma$ boundaries in lathy ferrite (weld 3) both labeled $A$. In the image carbides can be seen which have the elongated, lerticular morphology discussed above. Furthermore, two sets of linear defects can be seen within this boundary. One ser runs parallel to the carbides and are irregularly spaced. The second set are at an angle to the carbides and are regularly spaced.

No precipitation of carbides associated with extrinsic dislocations was seen on any of the boundaries studied.

\subsection{Phase Boundary Migration in Lathy Ferrite}

Examination of the welds with lathy ferrite microstructure indicated that substantial phase boundary migration nccurred during heat treatment at $550^{\circ} \mathrm{C}$ and $600^{\circ} \mathrm{C}$.

Figure 5-14 a) and b) show bright field images from samples of weld 3 aged for 24 hours at $550^{\circ} \mathrm{C}$. Most of the interphase carbides in these images are $>50 \%$ embedded in the austenite phase. Furthermore, most of the $\alpha / \gamma$ boundary segments that extend between interphase carbides bow out into the ferrite phase.

Figures 5-15 a) and b) show bright field images of samples of weld 3 aged for 6 and $\angle 4$ hours respectively at $600^{\circ} \mathrm{C}$. The boundaries in these images have migrated a sufficient amount to leave the carbides completely embedded in the austenite.

The composition of the austenite formed during the 24 hour heat-treatment at $600^{\circ} \mathrm{C}$ was measured using EDS. The probe was placed at point $A$ in Figure 5-15 b). 
The probe diameter $\approx 600 \AA$. The composition measured was $67.2 \mathrm{w} t \% \mathrm{Fe}, 18.8 \mathrm{wt} \%$ $\mathrm{Cr}$, and $13.3 \mathrm{wt} \% \mathrm{Ni}$. 
Table V-1

Measured Values of Chromium Depletion Widths $\left(W_{\alpha}\right)$

$\begin{array}{ccc}\text { Profile \# } & \begin{array}{c}\text { Aging time } \\ \text { (hours) }\end{array} & \begin{array}{c}W_{\alpha} \text { (measured) } \\ (A)\end{array} \\ 1 & 1.33 & 140 \\ 3 & 7.75 & 170 \\ 3 & 16.00 & 310\end{array}$



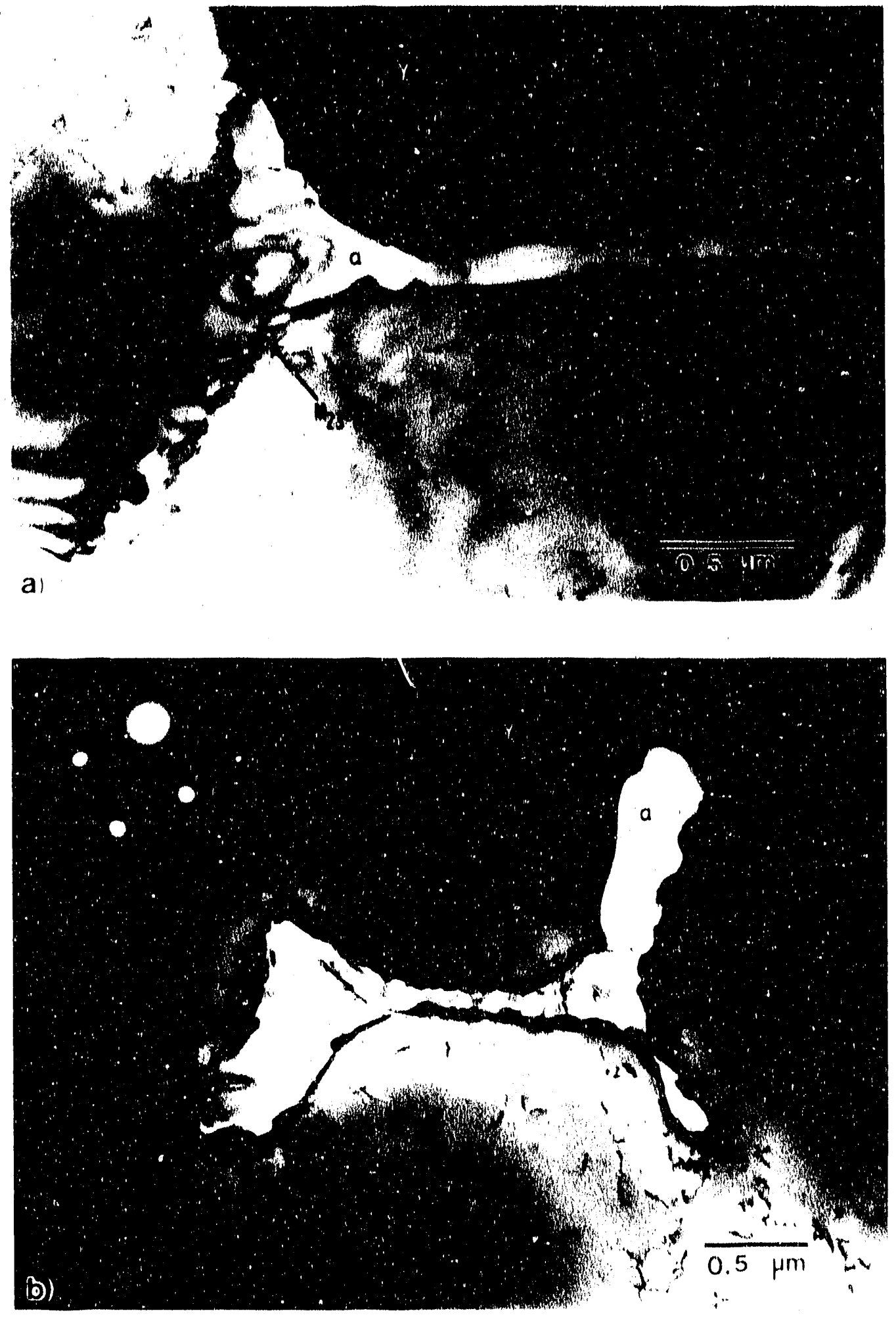

XBB $904-3010$

$5-1$ 


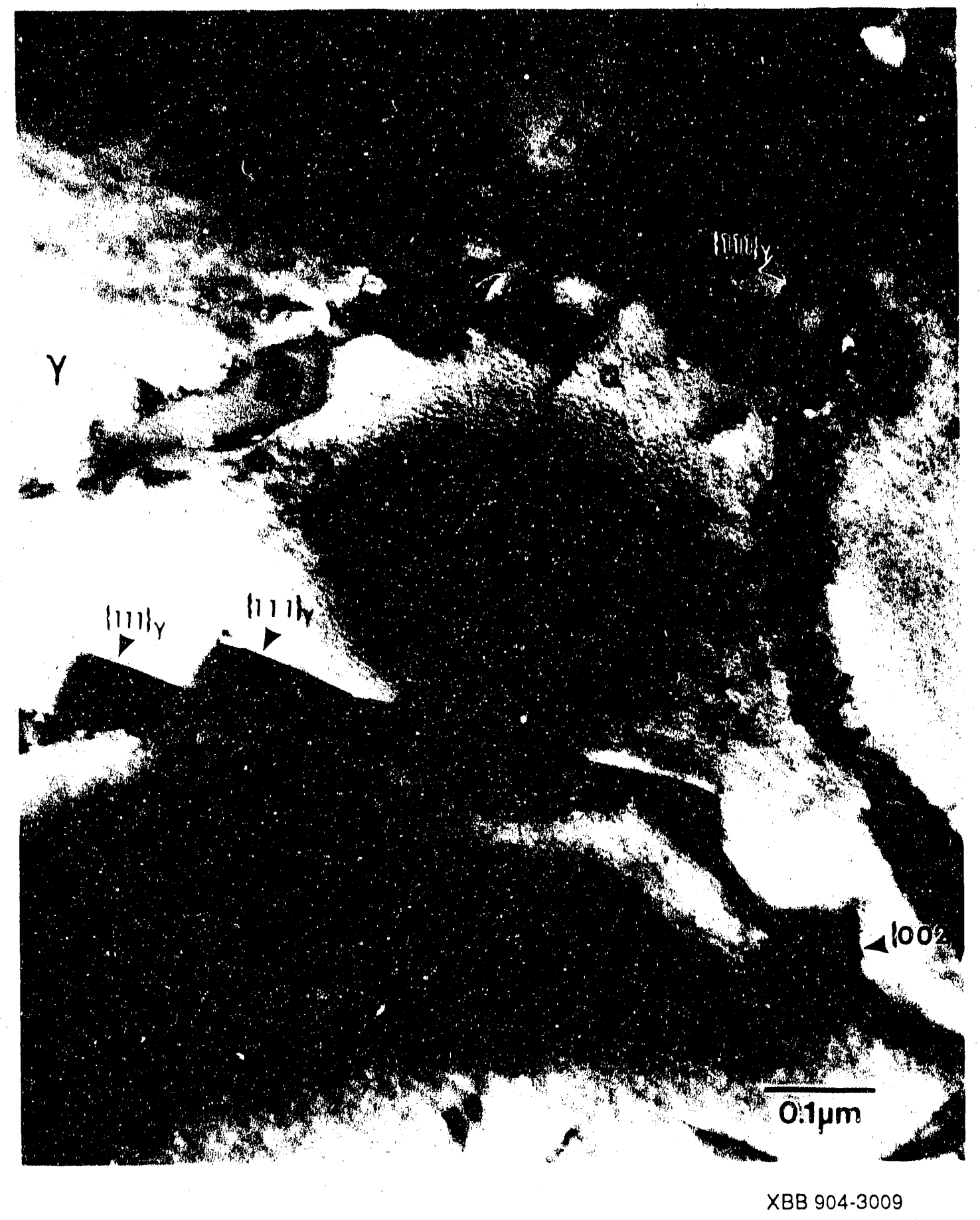





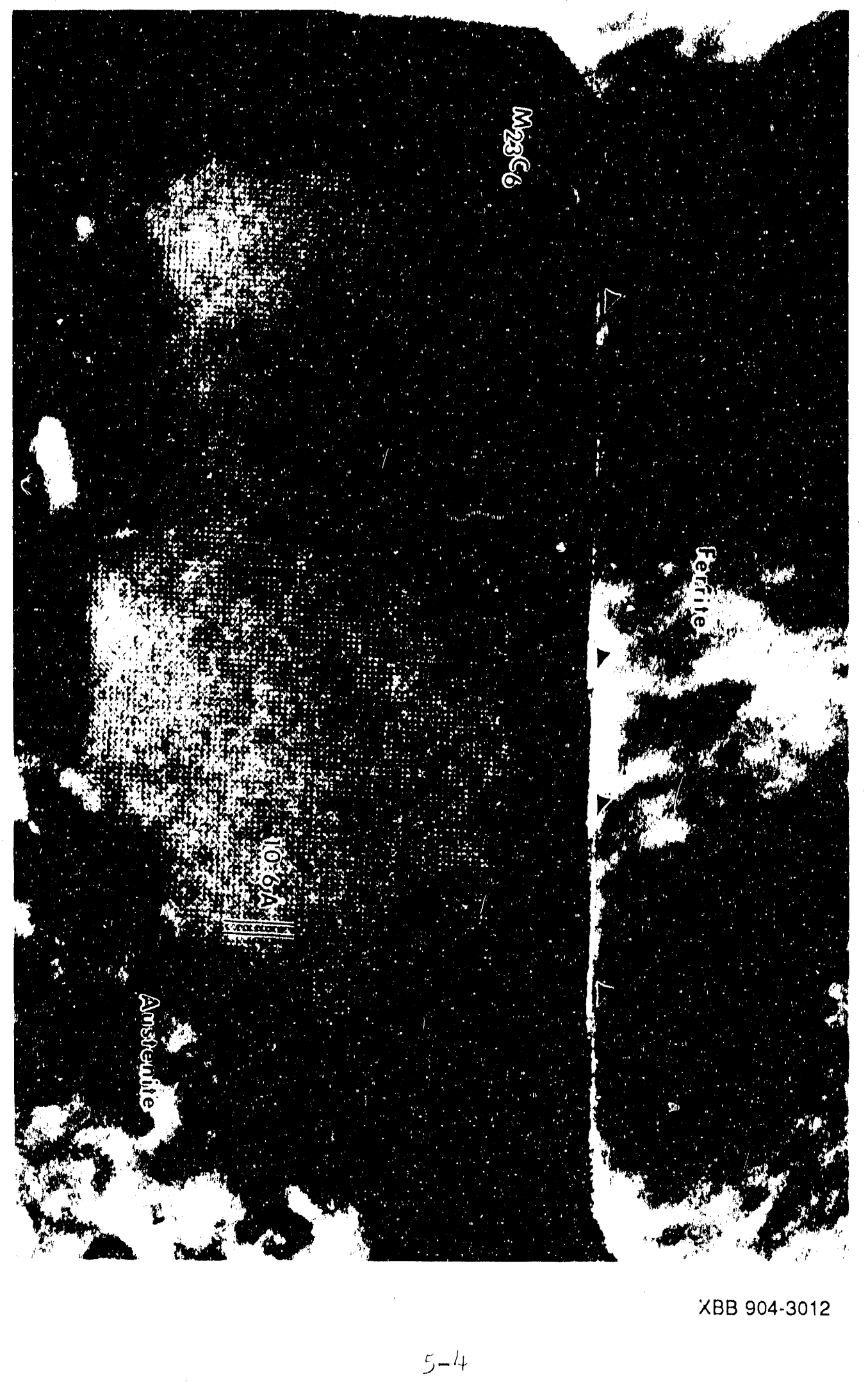




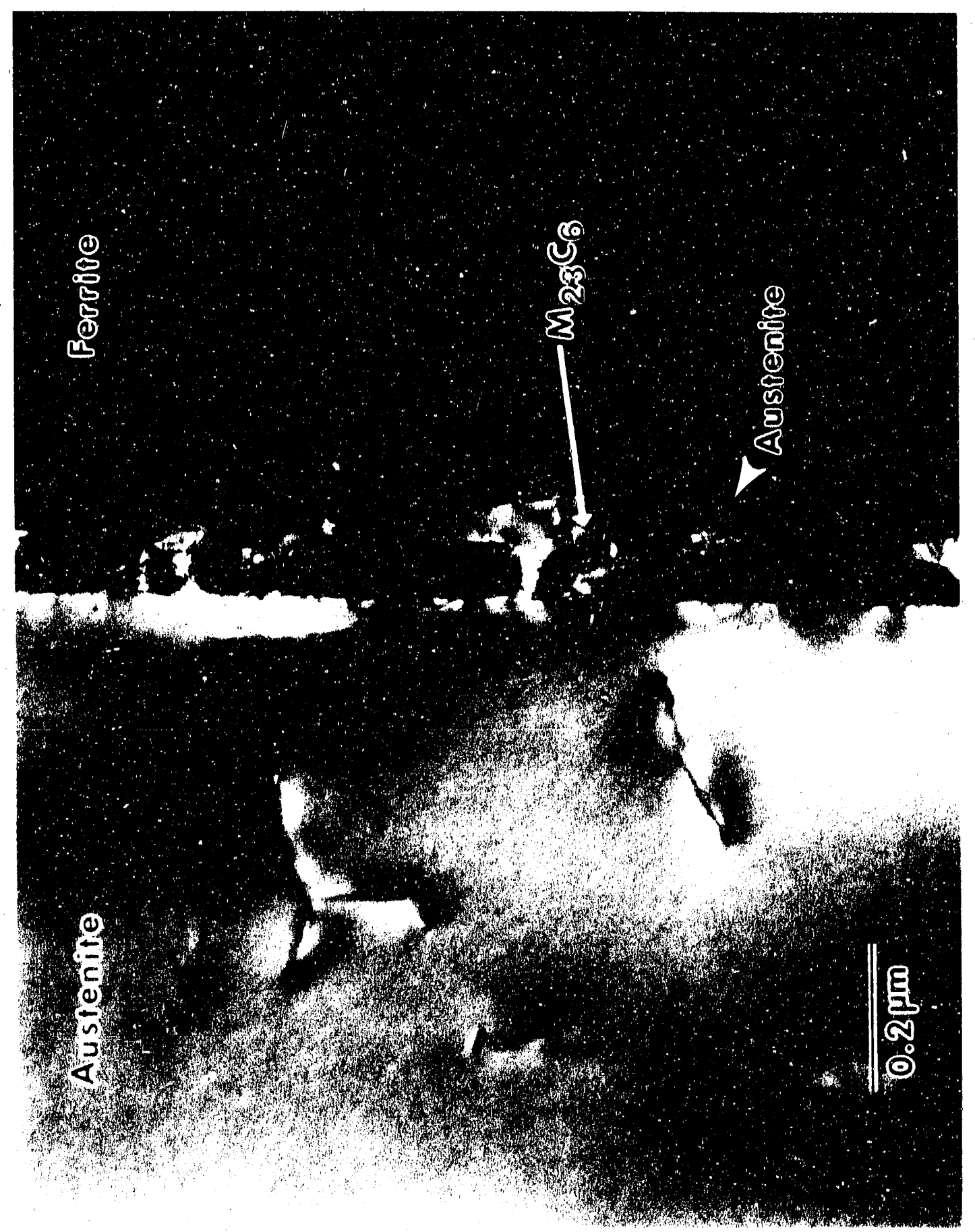

XBB $905-3812$ 

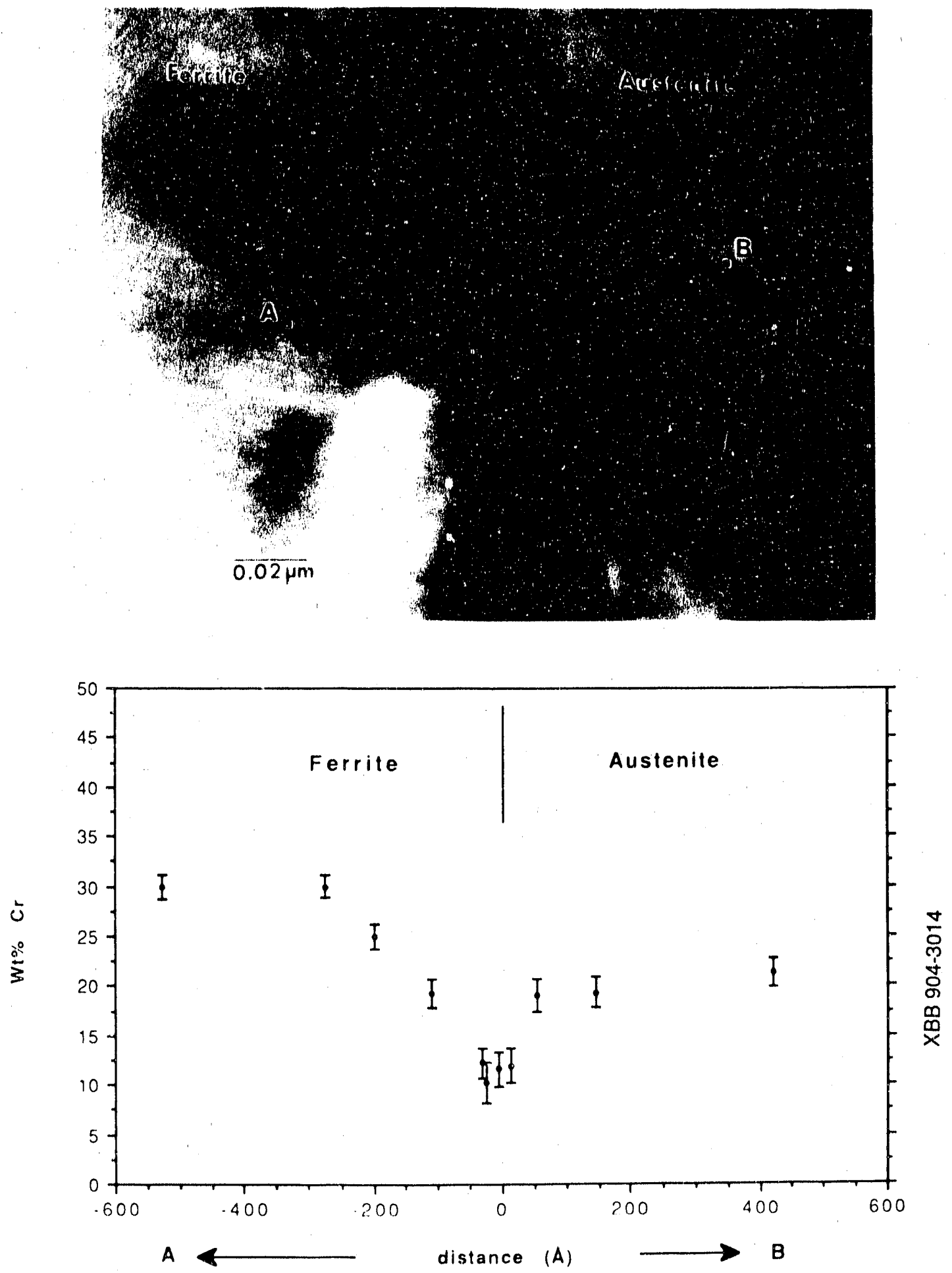

$5-6$ 

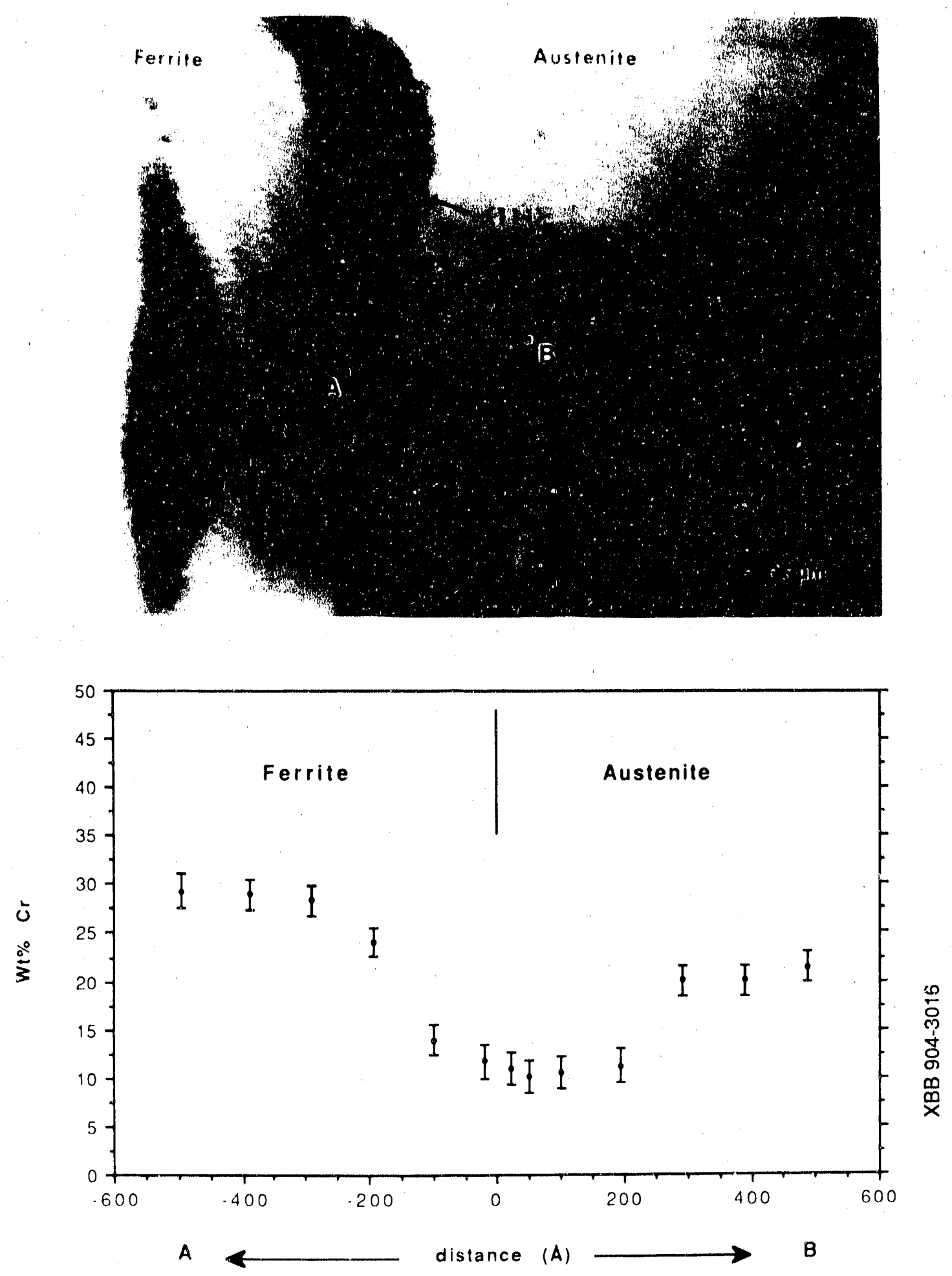

5-7 

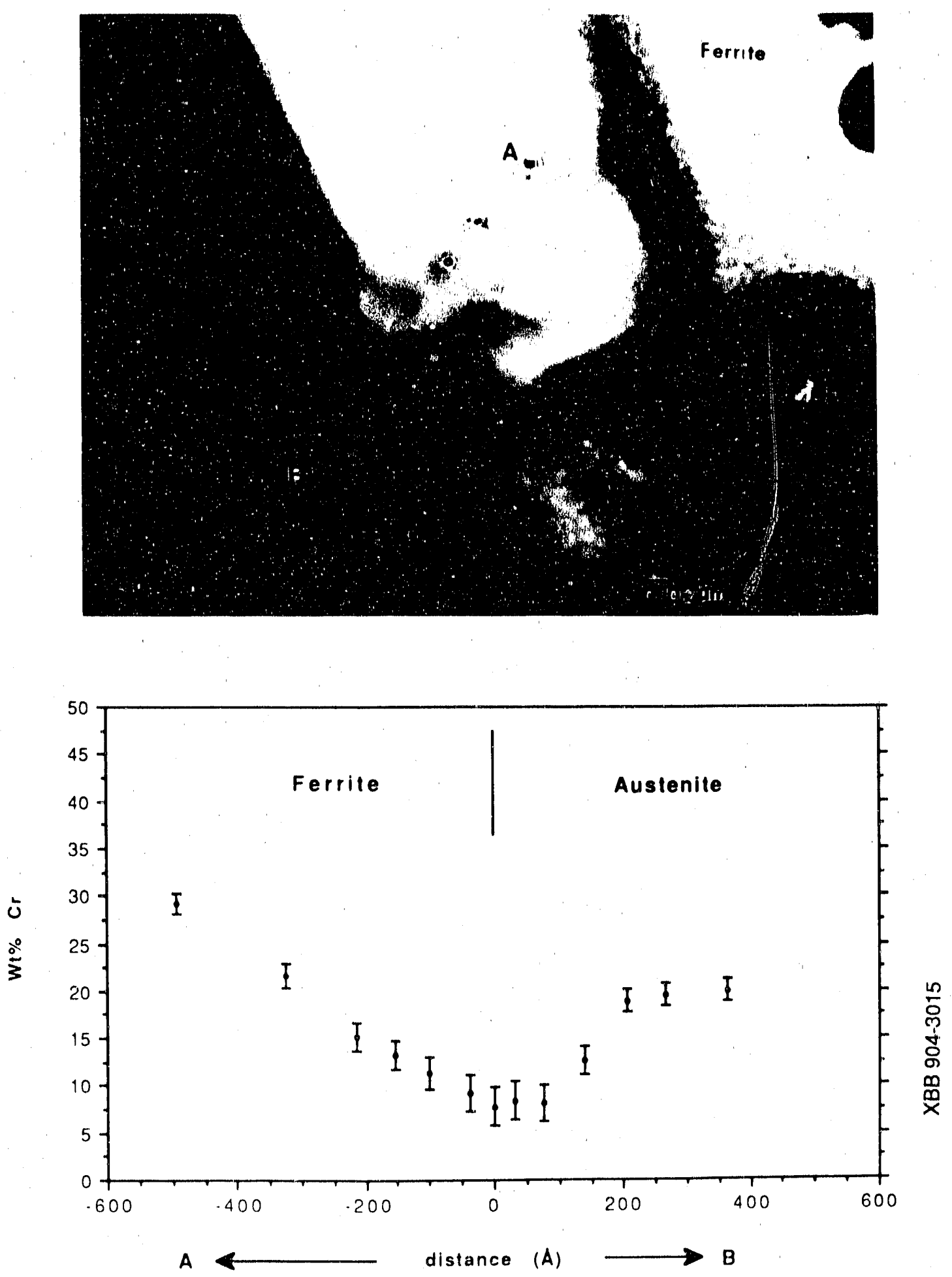

5-8 


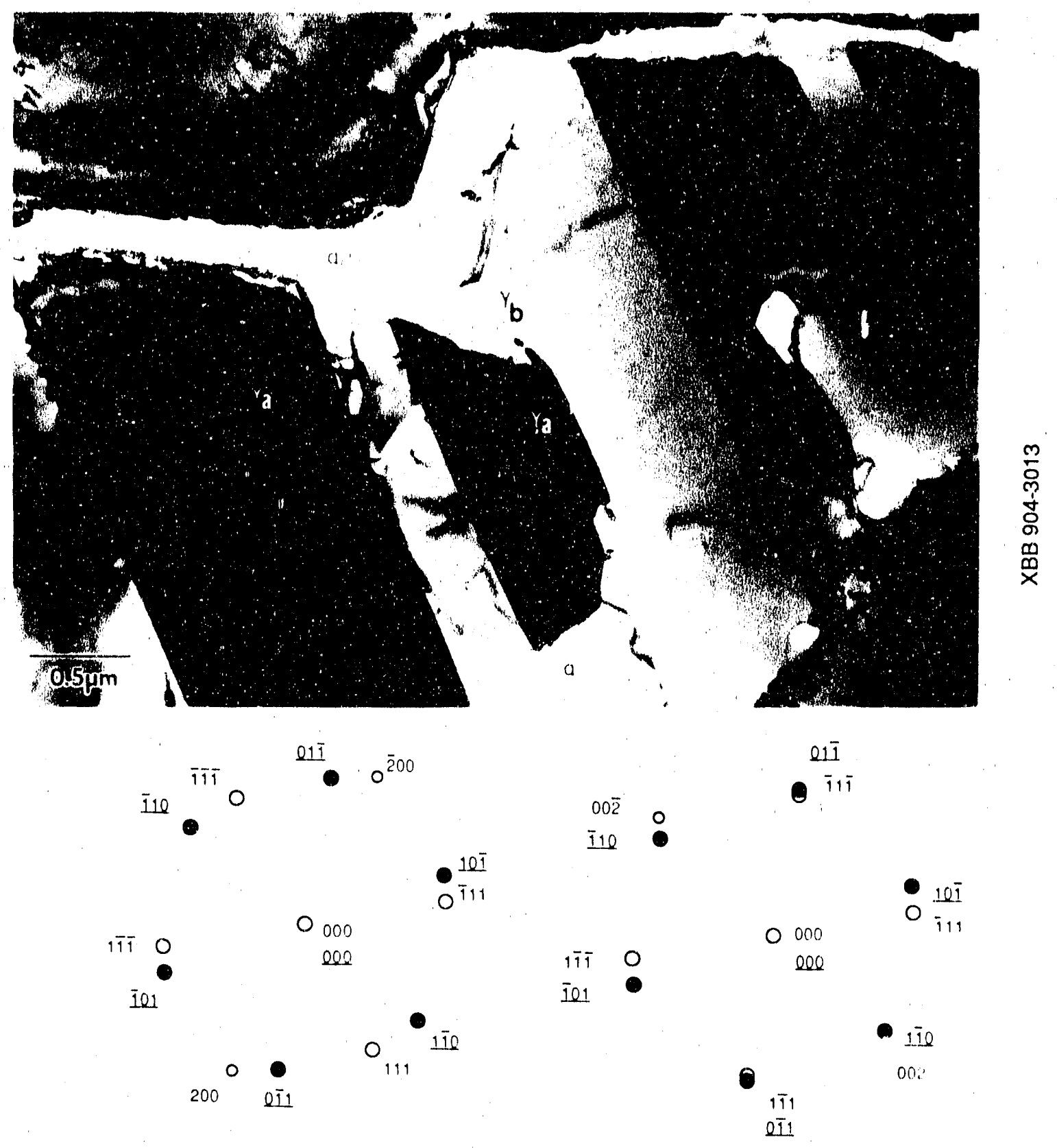

$$
\begin{aligned}
& -\because\lfloor 11\rangle_{(1 .} \\
& O=\mid 0 \overline{11}]_{\mathrm{ra}}
\end{aligned}
$$$$
\begin{aligned}
& 0=\left[111 \mathrm{~h}_{\mathrm{h}}\right. \\
& O=[\overline{1} \overline{1}]_{\mathrm{p}} \mathrm{p}
\end{aligned}
$$ 


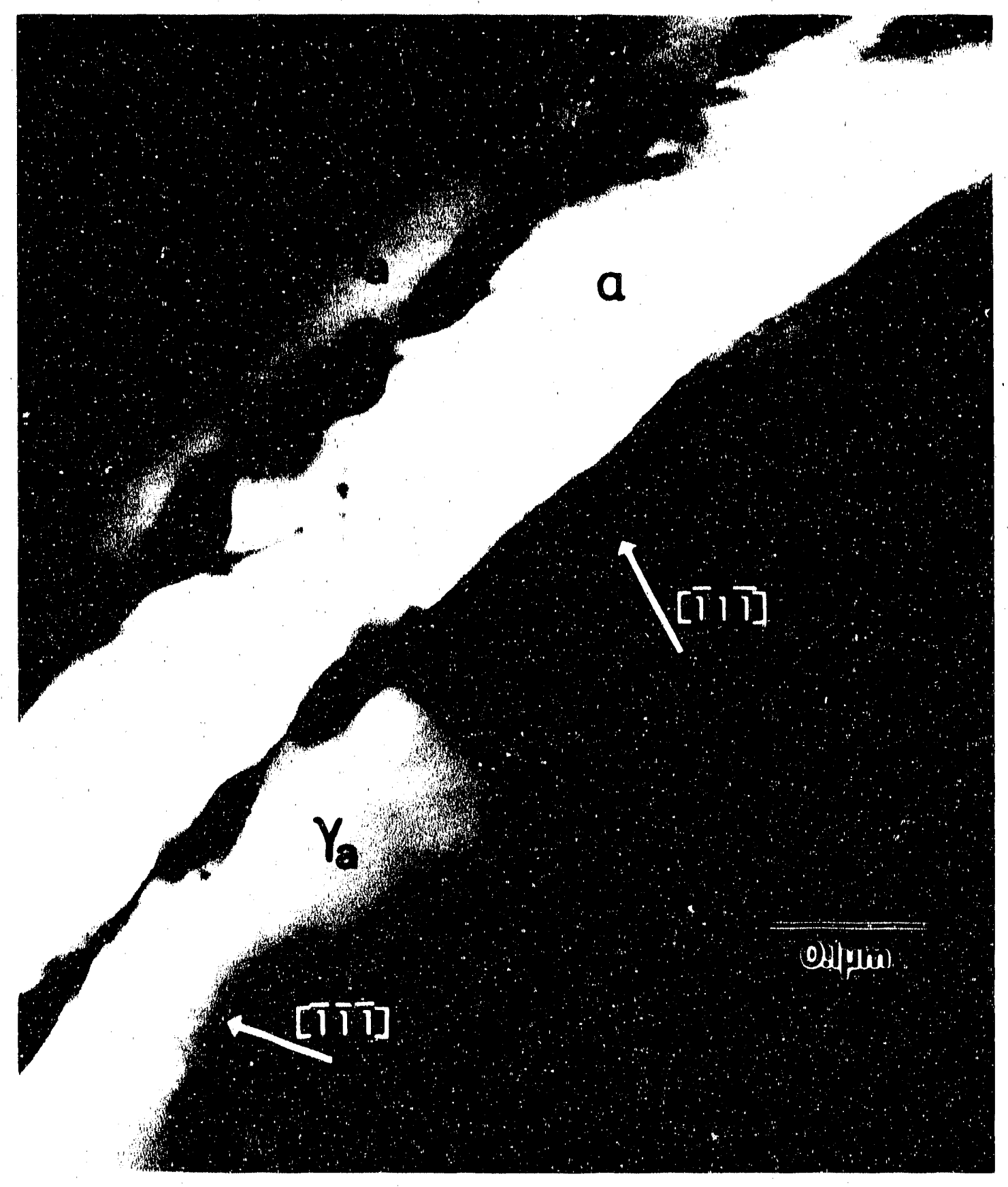

XBB 904-3008

\section{$5-10$}




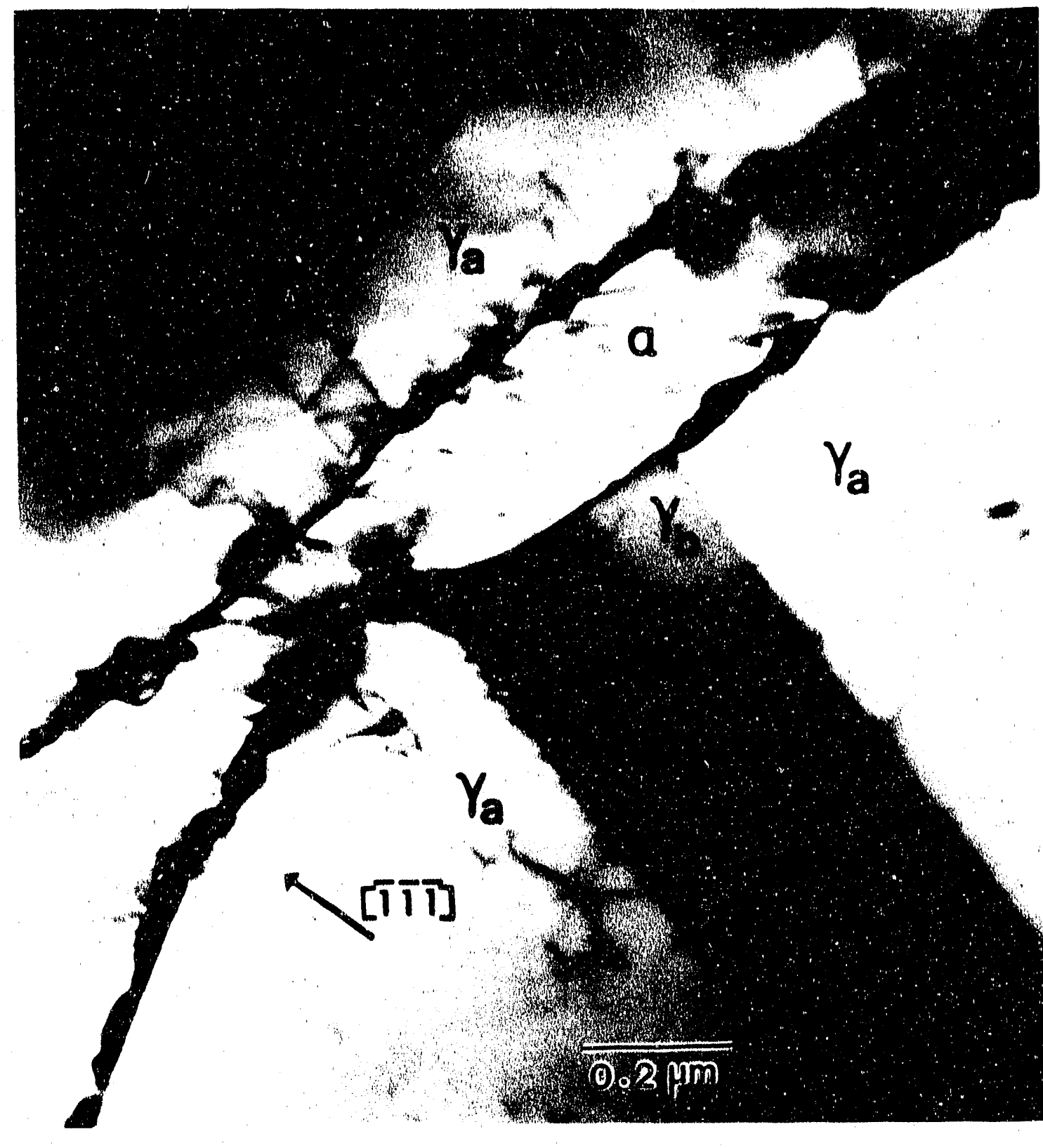

XBB 904-3007

5-11 

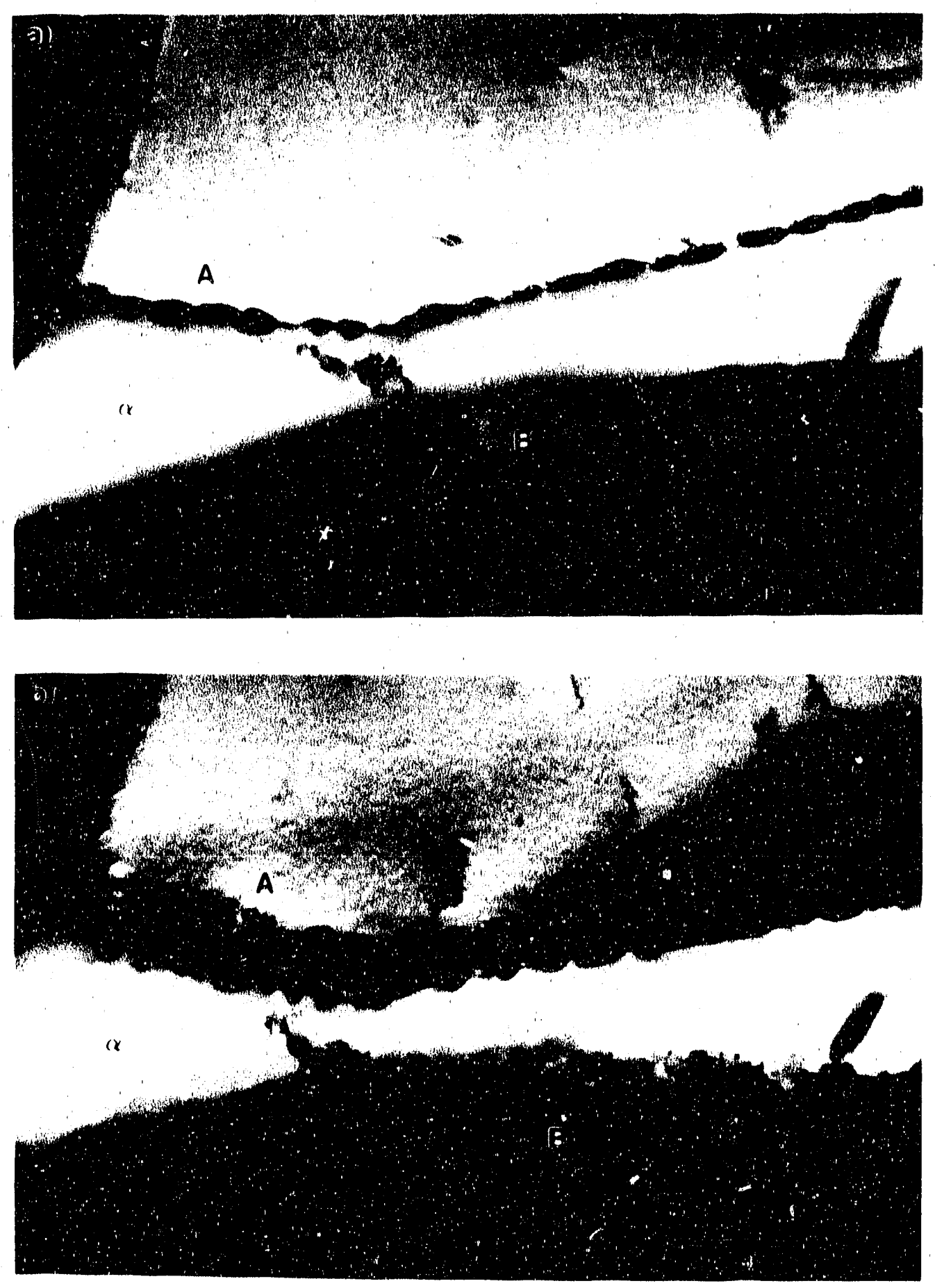

XBB 904-2837 


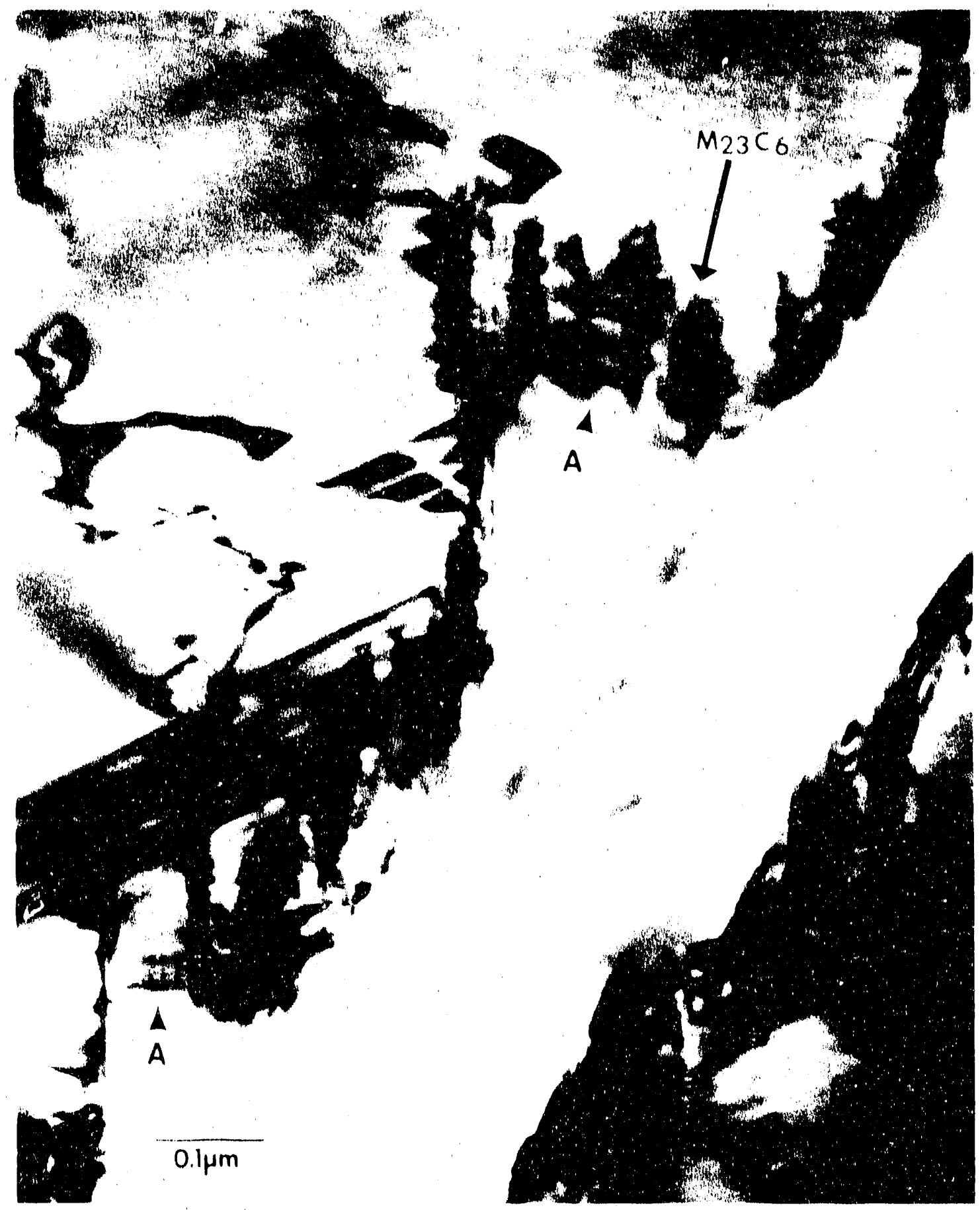

XBB 904-2834 

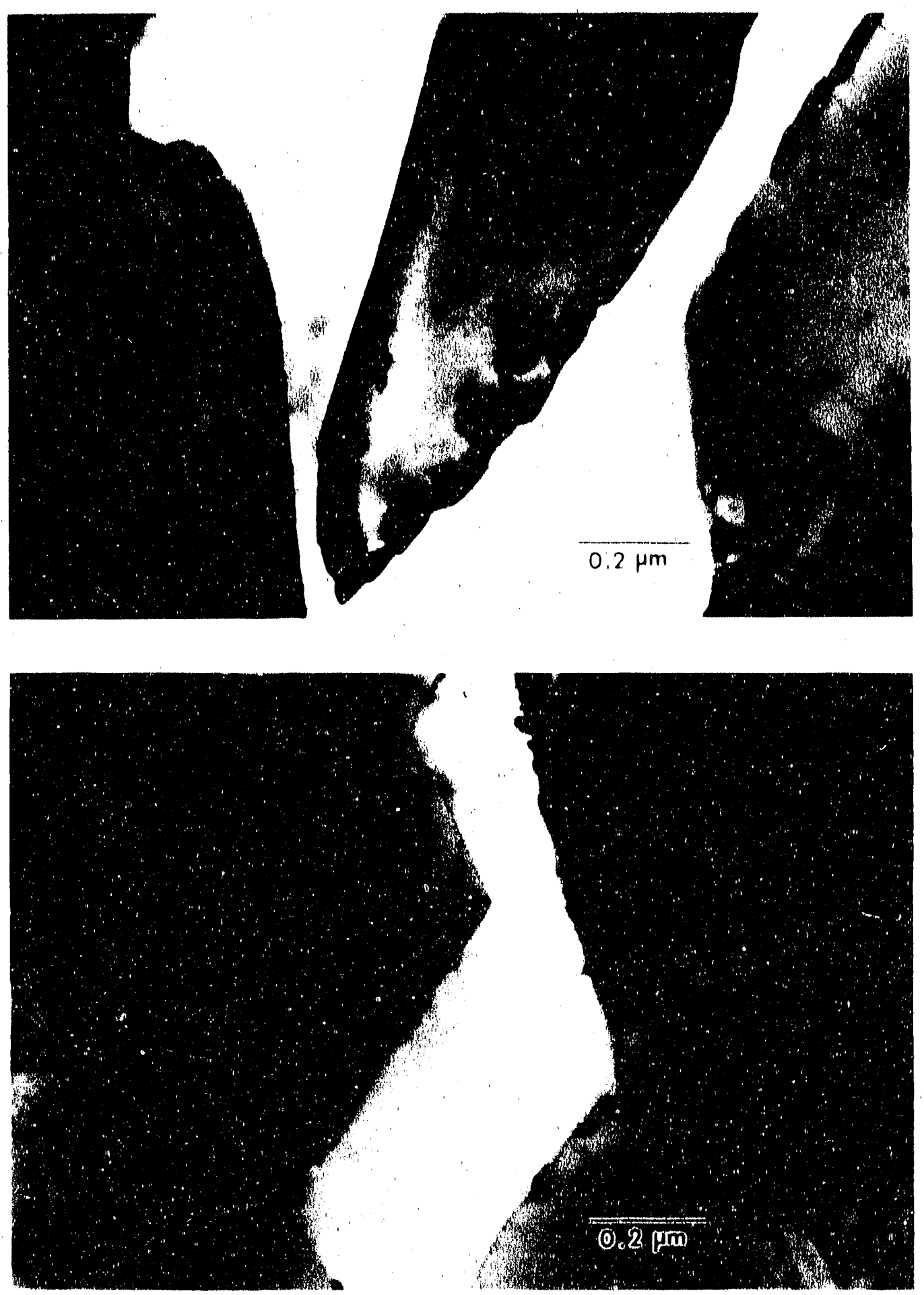

XBB 904-2833

$5-14$ 

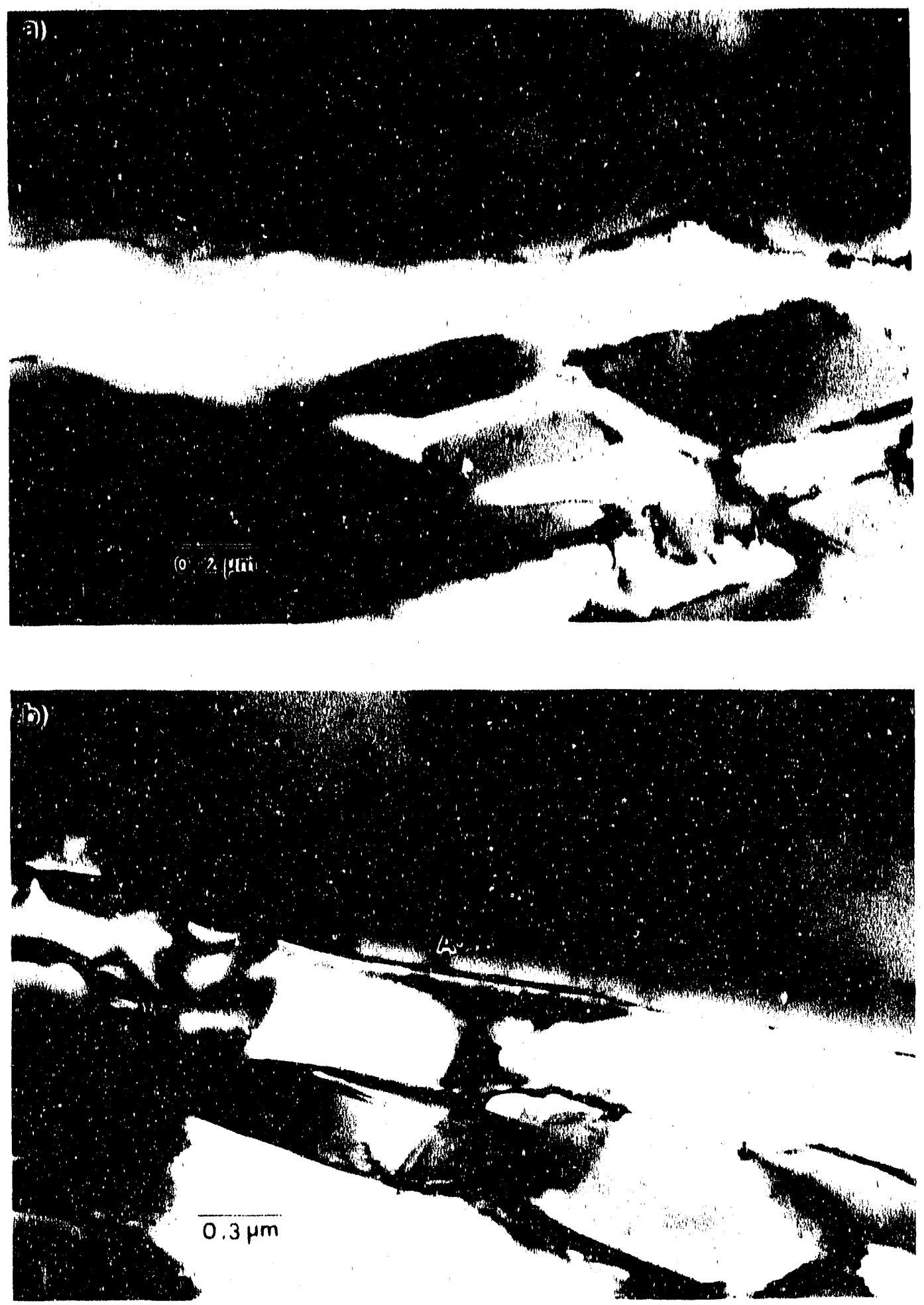

XBB 904-2836 


\section{Discussion}

\subsection{Carbide Precipitation in Skeletal Ferrite}

The results of transmission electron microscopy performed on samples of welds with skeletal ferrite which were heat-treated at $550^{\circ} \mathrm{C}$ for 1.33 to 96 hours indicate that most of the $\alpha / \gamma$ interfaces were high energy boundaries, although a few low energy iriterfaces (e.g. with the Nishiyama-Wasserman orientation $[41,42])$ were also seen. The high energy $\alpha / \gamma$ boundaries were generally covered with carbides, while the low energy interfaces supported a lower density of carbides. The carbides that precipitated on the interphase boundaries shared a cubellcube orientation relationship with the austenite phase as has been reported in previous studies [32-36].

That the carbides grew preferentially into the ferrite is presumably due to the fact that transport of chromium to the carbide is the rate controlling step in the growth of the carbide, and that the ferrite phase possesses a greater amount of cinromium than does the austenite phase. Consequently the flux of chromium to the carbide will be much greater from the ferrite phase than from the austenite phase.

Although there was no specific orientation relationship between the carbide and the ferrite phase, the carbides were nevertheless faceted. Initially it was thought that the facets corresponded to partially coherent ferrite/carbide interfaces. However, since no ferrite reflections were found parallel to the pole of the facet plane, there are no ferrite planes parallel to the carbide facet. The facet interface is not partially coherent, therefore, and an alternate explanation must be given for its planarity.

Crystals grown from the vapor phase show faceting on planes of high atomic density [46]. This is due to the fact that such planes possess low surface energies [46]. Because of the low energy of these planes, thickening of the crystals occurs by a ledge growth mechanism on these facets [47]. In the complex cubic siructure of the $\mathrm{M}_{23} \mathrm{C}_{6}$ carbides, the planes of densest packing are $\{111\}$ and $\{100\}$ [29]. Since these are the most common facet planes observed in the present study, it is suggested that these are low energy planes by virtue of their high atomic density as in crystals grown from the vapor. The growth ledges shown in Figure 5.4 support this interpretation.

The decomposition of the skeletal ferrite to austenite (e.g. in Figure 5-5) which initiates at the $\alpha / \gamma$ phase boundary in samples heat-treated for increasingly longer times at $550^{\circ} \mathrm{C}$ is not surprising in view of:

i) the fact that the ferrite phase is thermodynamically unstable at temperatures less than $\approx 1100^{\circ} \mathrm{C}$; 
i) the formation of a chromium depleted zone along the $\alpha / \gamma$ boundary lowers the stability of the ferrite phase further, since chromium is a ferrite stabilizer.

\subsection{Chromium Depletion Profiles}

One of the reasons for obtaining the chromium profiles was to determine the chromium concentration at the interphase boundary by fitting the profile to the solution of the appropriate diffusion equation and then extrapolating the fitted curve to the boundary. However, from the shape of the measured profiles and, in particular, from the flatness of the profile on the austenite side of the interphase boundary, it is clear that it is not possible to fit the profile in the austenite grain to a simple diffusion equation. This will be discussed at greater length below.

The shape of the chromium profile on the ferrite side of the interphase boundary appears compatible with an error function solution to the diffusion equation. Consequently $W_{\alpha}$ may be calculated from the diffusivity of chromium in the ferrite, using the expression $W=(D t)^{1 / 2}$ as discussed in section 1.3.2. The diffusivity values used were taken from Smith and Hales [48]. These authors used the standard serial sectioning technique to experimentally determine $D_{\alpha}$ and $D_{\gamma}$ in a duplex stainless steel $(18.5 \mathrm{wt} \% \mathrm{Cr}, 9.5 \mathrm{wt} \% \mathrm{Ni})$ over the temperature range $600^{\circ} \mathrm{C}-1000^{\circ} \mathrm{C}$. By extrapolation, values for $550^{\circ} \mathrm{C}$ are obtained. These values are:

$D_{\gamma}=2.2 \times 10^{-17} \mathrm{~cm}^{2} / \mathrm{sec}$ and $D_{\alpha}=1.96 \times 10^{-15} \mathrm{~cm}^{2} / \mathrm{sec}$.

The calculated values for $W_{\alpha}$ are shown in Table VI-1 along with the values obtained from the chromium profiles. One assumption that was made in obtaining the calculated values was that precipitation started at $:=0$. Consequently the calculated values are upper-bound estimates.

The measured values of $W_{\alpha}$ are substantially lower than the calculated values. It should be pointed out, however, that $D_{\alpha}$ has some experimental error associated with it i.e. $D_{\alpha}=1.96\{+9.5 / .1 .5\} \times 10^{-15} \mathrm{~cm}^{2} / \mathrm{sec}$. If the lower bound value is used to

calculate $W_{\alpha}$ a value of $150 \mathrm{~A}$ is obtained for profile \#1 which is quite close to the experimental value of $110 \mathrm{~A}$. Furthermore, as mentioned above, in calculating $W_{\alpha}$ it has been assumed that precipitation commences at $t=0$, which is of course not true for all boundaries, and which could explain, in part, why the measured values of $W_{\alpha}$ are less than the calculated values. Finally it is possible that the rate of carbide growth is not purely diffusion controlled, but is a combination of diffusion and interface control. The resulting chromium profile would be compatible with an effective diffusion coefficient which was smaller than the true value. Hence the measured $W_{\alpha}$ would be 
smaller than that calculated with the true value of $D_{\alpha}$. It is impossible to determine from the given data, which of these effects is actually operative.

In Figure 5-7, the flatness of the chromium profile in the austenite phase is most apparent although it is also clearly a feature in the profile presented in Figure 5 8. In fact, the size of this flat region in Figure 5-7 is so large that the width of the chromium depleted region in the austenite phase is obviously larger than that in the ferrite phase. This fact is clearly in variance with the much greater diffusivity of chromium in the ferrite phase relative to the austenite phase. In fact, the results suggest that something in addition to simple diffusion of chromium is responsible for the shape of the measured chromium profiles. It is hypothesized that the bright field image in Figure 5-7 showing the area from which profile 2 was obtained, indicates that $\alpha \rightarrow \gamma$ transformation has occurred at this boundary.

As discussed in the previous section, the chromium depletion will lower the stability of the ferrite phase. In fact after 24 hours at $550^{\circ} \mathrm{C}$, enough $\alpha \rightarrow \gamma$ transformation occurs to leave carbides in some areas completely embedded in the austenite (Figure 5-5). Even after shorter aging times there is evidence of $\alpha \rightarrow \gamma$ transformation in the vicinity of interface carbides. Figure 5-7 is an example. Notice that the top carbide is $\approx 50 \%$ embedded in the austenite. Furthermore it appears that austenite growth has occurred such that a (111) $\gamma \|(111) M_{23} C_{6}$ habit plane has been rraintained. This is significant in that this is known to be the plane of best-fit between the austenite and the carbide lattices as discussed in section 1.4.1. Similar $\{111\}$ habit planes can be seen on interface carbides in Figures 5-10 and 5-11. Here too it appears that phase boundary migration has occurred such that this habit plane is maintained.

Thus it is postulated that the unexpectedly large sica of the chromium depleted zone in the austenite phase relative to that in the ferrite phase is the result of the transformation of the chromium depleted zone in the ferrite phase to austenite which is epitaxially related to the existing austenite grain. As will be explained in section 6.5., the greater than anticipated width of the chromium depleted zone in the austenite phase is thought to be responsible for the intergranular corrosion susceptibility of weids with lathy ferrite microstructures.

The phase boundary migration which results from the $\alpha \rightarrow \gamma$ transformation, also accounts for the flatness of the chromium profile in Figure 5-7. Clearly this profile does not even approximate the solution of the diffusion equation illustrated in Figure 1-4.

Some $\alpha \rightarrow \gamma$ transformation may have occurred at the phase boundary from which profile \#3 was obtained (Figure $5-8$ ), as indicated by the flatness of the profile in the 
austenite. However, the amount of transformation is less than in profile \#2 despite the longer aging time. It is possible that this is due to the proximity of the two carbides in profile \#3 which act to pin the phase boundary. Another possible explanation is that there was no (111) plane of best-fit at a suitable angle to the phase boundary, as there was in Figure 5-7.

Analogous AEM studies in single phase austenitic stainless steels have yielded asymmetric chromium profiles across sensitized $\gamma / \gamma$ grain boundaries $[40,49]$. The origin of this asymmetry was determined to be grain boundary migration. The boundary migration was always of the parallel grain into the high-angle grain. Since the $\gamma / M_{23} C_{6}$ interface energy is lower between the carbide and the parallel grain, the driving force for grain boundary migration in the single phase austenitic steel was thought to be a reduction in the $\gamma / \mathrm{M}_{23} \mathrm{C}_{6}$ interface energy. This mechanism was observed to be similar to the Tu and Turnbull mechanism for cellular precipitation $[40,49]$, although in this case the volume of precipitate is too low to give a cellular morphology.

In summary, except for the possibility of carbide growth being partly interface controlled, the kinetic model of the formation of chromium depleted zones based on chromium diffusion is considered to be essentially correct. The relative magnitudes of $W_{\gamma}$ and $W_{\alpha}$ will vary however, depending on the amount of $\alpha \rightarrow \gamma$ transformation occurring at the boundary. The iransformation occurs because the depletion of chromium lowers the stability of the ferrite. This transformation appears to be energetically more favorable if a $\{111\}$ plane of best-fit between the carbides and the austenite can be maintained.

\subsection{Effect of Phase Boundary Misorientation}

Figures 5-10 and 5-11 show that precipitation occurred on the $\gamma_{\mathrm{a}} / \alpha$ interface but not the $\gamma_{b} / \alpha$ interface. This is attributed to the greater coherency along the $\gamma_{b} / \alpha$ interface. That is, since $\gamma_{a}$ and $\gamma_{b}$ are iwins, and since each forms an interphase boundary with the same grain of ferrite, the only distinction between the $\alpha / \gamma_{a}$ boundary and the $\alpha / \gamma_{b}$ boundary is that the $\alpha / \gamma_{a}$ boundary is a high energy boundary while the $\alpha / \gamma_{b}$ bcundary is a low energy bouridary. This graphically illustrates the role of boundary misorientation on carbide precipitation. Note that in Figure 5-10 the $\gamma_{b} / \alpha$ boundary is slightly curved, the boundary normal varying between the two coherent. crystallographic directions: 
$[\overline{11} \overline{1}]_{\gamma_{0}} \|[01 \overline{1}]_{\alpha}$

and

$$
[00 \overline{2}]_{\gamma_{b}} \|[\overline{1} 10]_{\alpha}
$$

It follows that the $\alpha / \gamma_{b}$ interface is partially coherent all along its length and, presumably, this precludes it from serving as a preferential site for carbide precipitation.

This result verifies that the degree of $\alpha / \gamma$ phase boundary misorientation and hence the phase boundary energy will affect the density of carbide precipitates at the boundary. Such an effect has been used elsewhere to explain the low carbide density existing on many boundaries in the lathy ferrite microstructure. This result justifies the use of an effective $N_{L}{ }^{\alpha-\gamma}$ value for the lathy ferrite microstructure as discussed in section 1.3.4.

\subsection{Carbide Nucleation Site in Lathy Ferrite}

From the above discussion it is clear that the partially coherent, low energy boundaries found in the lathy ferrite microstructure are not preferential sites for carbide precipitation. The carbide density in the lathy ferrite microstructure is indeed lower than in the skeletal ferrite microstructure [50].

The results presented in section 5.4. suggest that carbide precipitation within low energy interfaces occurs preferentially on some linear defect. The set of defects running parallel to the carbides in Figure 5.13 are thought to be ledge risers, since their uneven spacing is consistent with ledge growth. This is illustrated schematically in Figure 6-1. The second set of defects are more likely intrinsic dislocations. The spacing of intrinsic dislocations will be regular since it is governed by the degree of misfit between the two lattices. Since the carbides are parallel to the ledge risers, these must be the preferred nucleation sites within low energy boundaries.

These results differ from those of Southwick and Honeycombe [33] who observed precipitation on both intrinsic dislocation arrays and extrinsic dislocations as well as ledge risers. However these authors aged their samples at higher temperatures $\left(700^{\circ} \mathrm{C}-880^{\circ} \mathrm{C}\right)$ at which the ledge density is expected to be smaller [20].

In summary, the low energy boundaries existing in the lathy ferrite microstructure provide fewer nucleation sites for carbide precipitates. The carbides which do precipitate on these boundaries do so preferentially on ledge risers. 


\subsection{Effect of Phase Boundary Migration in Lathy Ferrite}

As discussed in section 1.3.4., the lathy microstructure satisfies the microstructural criterion for immunity to sensitization. Yet from Figure $1-5$ it is clear that this microstructure is sensitized at $550^{\circ} \mathrm{C}$. It was proposed that since many of the $\alpha / \gamma$ boundaries in this microstructure are low energy boundaries, the density of carbide precipitation along the interphase boundaries will be small, yielding an effective $N_{L}{ }^{\alpha-\gamma}$ value which no longer satisfies the criterion for immunity. The results discussed in the last two sections indicate that low energy boundaries do not have a high density of carbide precipitates. Nevertheless, TEM analysis shows that $\approx 50 \%$ of the boundaries have a sufficient density of carbides after a heat-treatment of 24 hours at $550^{\circ} \mathrm{C}$ for them 10 be included in the effective $N_{L}{ }^{\alpha-\gamma}$ value [50]. Calculating the effective $N_{L}{ }^{\alpha-\gamma}$ value to be $50 \%$ of the actual $N_{L} \alpha-\gamma$, yields a value which is still high enough for the microstructure to be immune to intergranular corrosion. How can the sensitization of this microstructure by heat-treatments at $550^{\circ} \mathrm{C}$ be explained?

It was observed that many of the interphase boundaries in the lathy microstructure have migrated as a result of some $\alpha \rightarrow \gamma$ transformation, leaving carbides partially embedded in the austenite as in Figure 5-14. As discussed in section 6.2., the effect of such a transformation is to produce a very wide chromium depleted zone in the austenite $\left(W_{\gamma}\right)$. Since the diffusion of chromium in austenite is slower than in ferrite, these boundaries are less rapidly healed. This could account for the sensitization susceptibility of lathy ferrite at $550^{\circ} \mathrm{C}$. That is, if the boundaries had not migrated, the chromium depleted zones on the austenite side of the interface would have been replenished by diffusion of chromium from within the adjacent region of ferrite. Instead, when the chromium depleted zone within the ferrite transforms to austenite, the result is the formation of a very wide chromium depleted zone within the austenite. This requires additional transport of chromium for healing to occur and could account for the unexpected susceptibility of welds with lathy ferrite to sensitization at $550^{\circ} \mathrm{C}$. The next question is why is the sensitization less severe at $600^{\circ} \mathrm{C}$ ? Of course the equilibrium concentration of chromium at the carbide interface- $\left(X_{C r}\right)^{\gamma}$-will be higher at $600^{\circ} \mathrm{C}$ than at $550^{\circ} \mathrm{C}$ due to the greater solubility of carbon in austenite at $600^{\circ} \mathrm{C}$. Using the thermodynamics data given in reference [24] for $600^{\circ} \mathrm{C}$ yields $\left(X_{C r}\right)^{\gamma}=5.5 w t \%$ which is only slightly higher than the value at $550^{\circ} \mathrm{C}(4.3 \mathrm{wt} \%)$ and well below the value required for passivation $(\approx 13 w t \%)$. This is a small effect therefore and can not account for the difference in sensitization behavior at these two temperatures. The 
diffusivity of chromium will also be higher at $600^{\circ} \mathrm{C}$. This accounts for the increased kinetics of sensitization and healing but does not account for the less severe sensitization at $600^{\circ} \mathrm{C}$.

Figure $5-15 \mathrm{a}$ shows that after aging for 6 hours at $600^{\circ} \mathrm{C}$ the $\alpha / \gamma$ phase boundary has migrated a sufficient amount to leave the carbides completely embedded in the austenite. Once the phase boundary has left the carbides completely behind, the carbide growth rate will slow since the chromium must all come from the austenite phase in which the diffusivity of chromium is low. Since after times as short as 6 hours, the carbides are completely embedded, the depletion zone should not increase substantially after this time. In addition, once neighboring carbides are no longer connected by a high diffusivity path such as the $\alpha / \gamma$ interphase boundary, a continuous path of chromium depletion will no longer exist. Because of the very high diffusivity of chromium along the $\alpha / \gamma$ interphase boundaries these boundaries form paths of approximately uniform activity of chromium, the activity being that which exists at the carbide/matrix interface. Consequently the interphase boundaries could form continuous paths of chromium depletion even though they are not $100 \%$ covered by carbides. When the carbides are no longer connected to a phase boundary, chromium that is present in the intercarbide space will not be rapidly transported to the growing carbides by short-circuit diffusion along an interphase boundary. The continuous chromium depleted zone will therefore be replaced by isolated chromium depleted regions about each carbide.

Once the $\alpha / \gamma$ boundary has migrated through the chromium depleted region in the ferrite phase, the austenite which forms from subsequent transformation of the ferrite will nol have a particularly low chromium content [51]. This is confirmed by the results of the AEM analysis which indicated that the newly formed austenite had a chromium content of $18 w t \%$. The migration of the $\alpha / \gamma$ interphase boundaries in the lathy ferrite microstructure is similar to that which occurs in the wrought duplex alloy containing $25 w t \% \mathrm{Cr}$ and $6 \mathrm{wt} \% \mathrm{Ni}$, as reported by Tedmon and Vermilyea [52].

An explanation for the lack of sensitization at $600^{\circ} \mathrm{C}$ can now be given. At $600^{\circ} \mathrm{C}$ the $\alpha \rightarrow \gamma$ transformation is more rapid than at $550^{\circ} \mathrm{C}$. After relatively short aging times the phase boundary has left the carbides behind. Once the boundaries are separated from the carbides continuous paths of chromium depletion no longer exist and the alloy is not susceptible to localized corrosion. 
Table VI-1

Measured and Criculated values of $W_{\alpha}$

$\begin{array}{cccc}\text { Profile \# } & \begin{array}{c}\text { Aging time } \\ \text { (hours) }\end{array} & \begin{array}{c}W_{\alpha} \text { (measured) } \\ (\AA)\end{array} & \begin{array}{c}W_{\alpha}^{\prime} \text { (calculated) } \\ (\AA)\end{array} \\ 1 & 1.33 & 140 & 305 \\ 2 & 7.75 & 170 & 739 \\ 3 & 16.00 & 310 & 1065\end{array}$




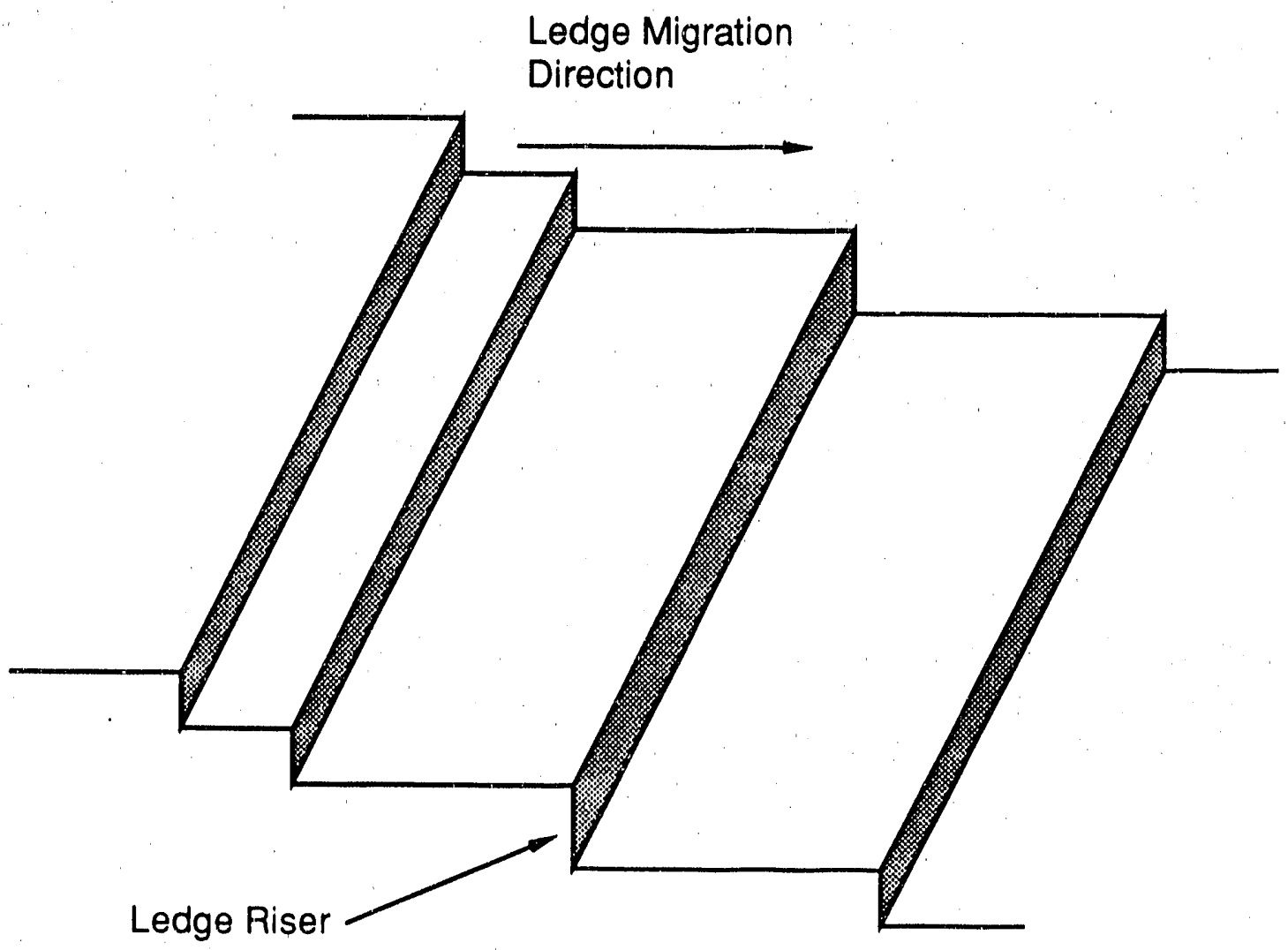

Figure 6-1 


\section{Conclusions}

The following conclusions are drawn from these results:

1) The kinetic model of the formation of Cr-depleted zones based on the diffusivities of chromium in the austenite and ferrite phases is essentially correct. However the widths of the depletion zones in the two phases also depends on the amount of $\alpha \rightarrow \gamma$ transformation that occurs during the carbide growth. The energetics of this transformation are more favorable if a plane of "best-fit" between a carbide the newly formed austenite can be maintained. This transformation results in a wide chromium depleted zone on the austenite side of the $\alpha / \gamma$ interface.

2) Carbide precipitation rate varies with misorientation of the $\alpha / \gamma$ boundary. The precipitation rate is lower for low-energy, partially coherent $\alpha / \gamma$ boundaries. Within the low energy boundaries carbides nucleate preferentially on ledge risers. Despite this precipitation behavior; approximately $50 \%$ of the boundaries in the lathy microstructure studied had significant carbide precipitation.

3) In the lathy ferrite microstructure $\alpha \rightarrow \gamma$ transformation occurs on many boundaries. This is thought to account for the sensitization of the lathy ferrite at $550^{\circ} \mathrm{C}$. From conclusion (1) we know that boundaries which have migrated during sensitization possess wide chromium depleted zones in austenite. Greater $W_{\gamma}$ values result in longer healing times since chromium diffusion in austenite is sluggish. At higher sensitization temperatures, $\alpha \rightarrow \gamma$ transformation leaves the carbides completely embedded in the austenite. When the carbides are separated from the phase boundary their growth rate slows, and upon further chromium diffusion continuous chromium depleted zones are replaced by isolated zones about each carbide. Since this occurs before 6 hours at $600^{\circ} \mathrm{C}$, the $\mathrm{Cr}$ depleted zones reach their maximum after short aging times. Further $\alpha \cdots \gamma$ transformation occurs without chromium depletion, the driving force being the partitioning of chromium and nickel to the ferrite and austenite respectively. 


\section{References}

1) P. Payson, Irans. AlME. 100, 306 (1932).

2) L. B. Pfeil and D. G. Jones, J. Lron Steel_Lnstu Lenden 128, 337 (1933).

3) V. R. Scherer, G. Reidrich and G. Hoch, Arch. Eisenhutten Wes, 13, 1 (1939).

4) T. M. Devine, Jeurnal of Electrochemical Soc., 126, 374 (1979).

5) T. M. Devine, Met. Trans. 11A, 791 (1980).

6) T. M. Devine, Acta. Met. 36, 1491 (1988).

7 ) "Encyclopedia/Handbook of Materials, Parts, and Finishes", p.447, Technomic, Westport, Ct, 1976

8 ) T. Takalo, N. Suutala, and T. Moisio, Met. Trans, 7A, 1591 (1976).

9 ) N. Suutala, T. Takalo, T. Moisio, Met. Trans. 10A, 1183 (1979).

10) N. Suutala, T. Takalo, T. Moisio, Met Trans. 10A, 512 (1979).

11 ) T. Takalo, N. Suutala, and T. Moisio, Met. Trans. 10A, 1173 (1979).

12) N. Suutala, T. Takalo, T. Moisio, Met. Trans. 11A, 717 (1980).

13) J.C. Lippold and W.F. Savage, Weld_ L_, 59(2), 48-s, (1980).

14) S.A. David, Weld. 1 , 60(4), 63-s, (1981).

$15)$ H. Menendez, J.S. Chen, and T.M. Devine, "Influence of Composition on the microstructure and sensitization of 308 Duplex Stainless Steel Welds", EPRI report.

16) S.A. David and G.M. Goodwin, "Solidification Behavior of Type 308 Stainless Steel Filler Metal"

$17)$ S.A. David, G.M. Goodwin, and D.N. Braski, Weld_ ل, 58(11), 330-s (1979).

18) G.L. Leone and H.W. Kerr, Weld. L. $_{1} 61(1), 13-\mathrm{s}$ (1982).

$19)$ G. Kurjumov and G. Sachs, Z. Phys, 64, 325 (1930)

$20)$ P.D. Southwick and R.W.K. Honeycombe, Mel. Sci., 14, 253 (1980)

21 ) J.A. Brooks, J.C. Williams, and A.W. Thompson, Met. Trans, 14A, 1271 (1983).

22 ) J. Singh, G.R. Purdy, and G.C. Weatherly, Met Trans. 16A, 1363 (1985).

23 ) C.E. Lyman, Weld Lل 58(7), 189-s (1979).

24) T. M. Devine, "Mechanism of Sensitization of Austenitic Stainless Steel" submitted for publication in corrosion science.

25 ) J. Crank, "The Mathematics of Diffusion", 2nd Ed., p.32, Oxford University Press, London (1975)

$26)$ J. S. Chen, M.S. Thesis, University of California Berkeley, Dept. of Materials Science and Mineral Engineering, 1989

27 ) ASTM Specification A262E-70 Test. 
28) M. H. Lewis and B. Hattersley, Acta Met, 13, 1159 (1965)

$29)$ F. R. Beckitt and B. R. Clark, Acta Mot, 15, 113 (1967)

$30)$ L. K. Singhal and J. W. Martin, Irans, of the AlME, 242, 814 (1968)

31) J. P. Adamson and J. W. Martin, Acta Met, 19, 1015 (1971)

32 ) H. D. Solomon, General Electric Technical Information Series, \#76CRD188 (1977)

33 ) P.D. Southwick and R.W.K. Honeycombe, Met. Sci, 16, 475 (1982)

34 ) J. Foulds and J. Moteff, Met..Irans, 13A, 173 (1982).

35 ) J. M. Vitek and S. A. David, Weld. لـ,

36) J. M. Vitek and S. A. David, Met. Trans, 18A, (1987).

$37)$ D. Williams, "Practical Analytical Electron Microscopy in Materials Science", p. 84, Philips Electron Instruments, inc. Electron Optics Publishing Group, Mahwah, New Jersey 1987.

38 ) E.L. Hall, D. Imeson, and J.B. Vande Sande, Phil. Mag.A, 43, 1569 (1985).

39 ) L. Reimer, "Transmission Electron Microscopy-Physics of Image Formation and Microanalysis", 2nd Ed.,p.387, Springer-Verlag, Berlin 1989

$40)$ E.L. Hall and C. L. Briant, Met. Irans, 15A, 793 (1984).

$41)$ Z. Nishiyama, Sci. Rep. Res. Inst Tohoku Univ, 23, 617 (1934).

42 ) G. Wasserman, Arch, Eisenhuten Wes., 16, 647 (1933).

43 ) J. B. VanderSande, A.J. Garratt-Reed, Y.M. Chiang, and T. Thorvaldsson, Uliramicroscopy, 14, 65 (1984).

44 ) J. A. Brooks, A.W. Thompson, and J.C. Williams, Weld. J., 63, 71-s (1984).

$45)$ H. Kokawa, T. Kuwana, and A. Yamamoto, Weld لـ, 92-s, March (1989).

$46)$ D.A. Porter and K.E. Easterling, "Phase Transformations in Metals and Alloys", p. 112, VNR International, Berkshire, England 1981.

47 ) lbid, p.180

48 ) A.F. Smith and R. Hales, Met. Sci, 10, 418 (1976).

49 ) T. A. Mozhi et al, Scripta Met, 20, 723 (1986).

$50)$ T.M. Devine and K. Mon, unpublished research.

51 ) J.M. Vitek and S. A. David, Scripta Mel., 19, 23 (1985).

$52)$ C.S. Tedmon and D.A. Vermilyea, Met. Trans, 1A, 2043 (1970). 

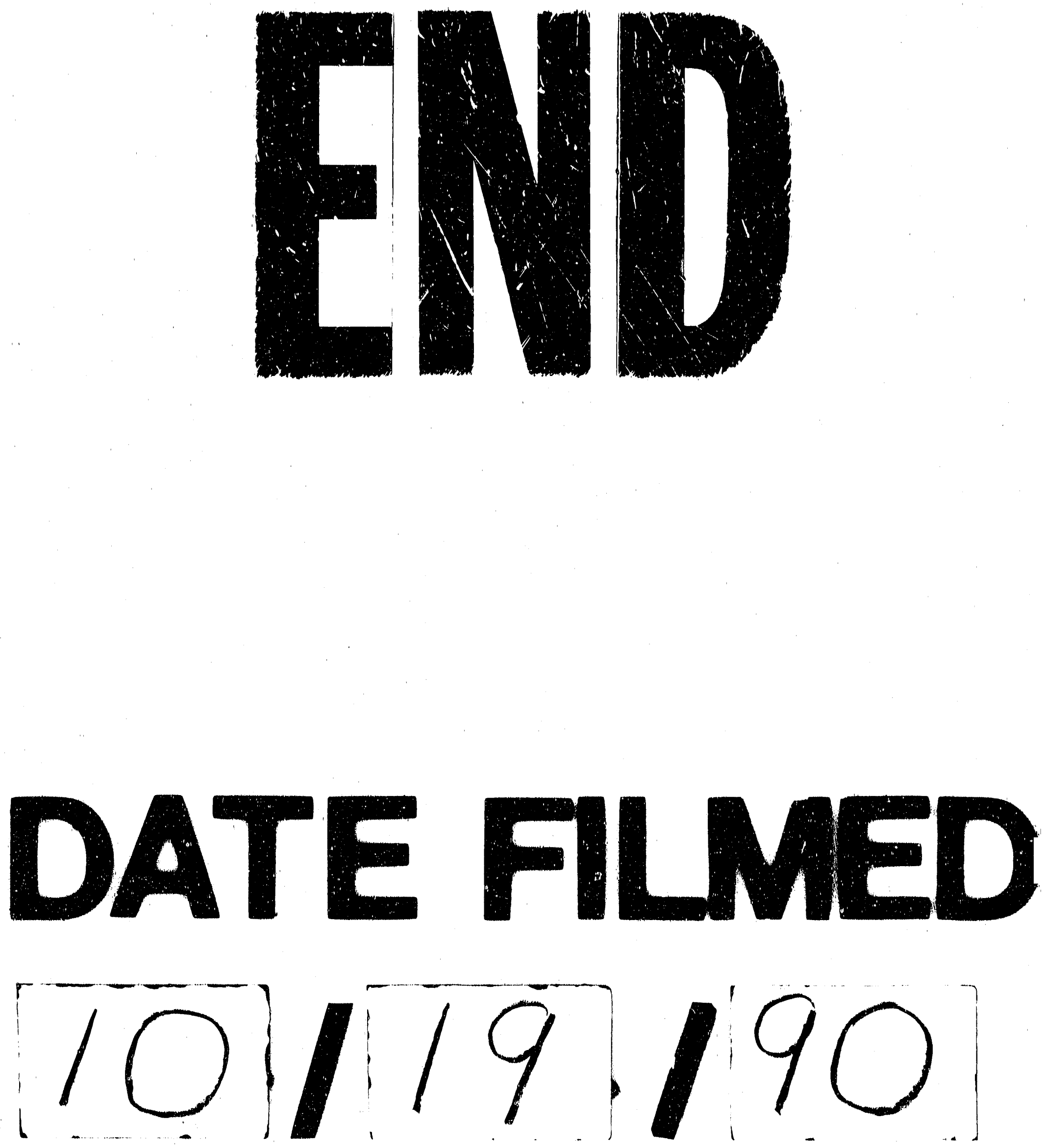
